\title{
Surface roughening and rippling during plasma etching of silicon: Numerical investigations and a comparison with experiments
}

\section{$\operatorname{AUTHOR(S):~}$}

Tsuda, Hirotaka; Nakazaki, Nobuya; Takao, Yoshinori; Eriguchi, Koji; Ono, Kouichi

\section{CITATION:}

Tsuda, Hirotaka ... [et al]. Surface roughening and rippling during plasma etching of silicon: Numerical investigations and a comparison with experiments. Journal of Vacuum Science \& Technology B 2014, 32(3): 031212.

\section{ISSUE DATE:}

2014-05

URL:

http://hdl.handle.net/2433/193618

\section{RIGHT:}

Copyright 2014 American Vacuum Society. This article may be downloaded for personal use only. Any other use requires prior permission of the author and the American Institute of Physics. 


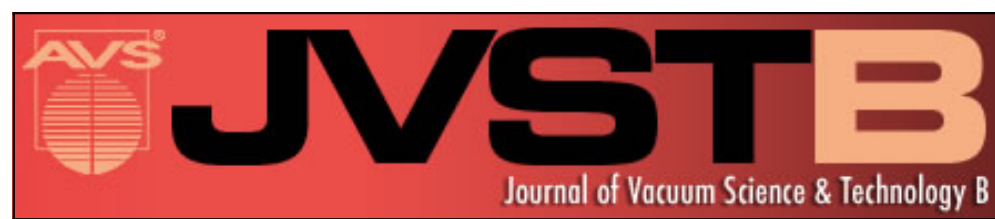

\section{Surface roughening and rippling during plasma etching of silicon: Numerical investigations and a comparison with experiments}

Hirotaka Tsuda, Nobuya Nakazaki, Yoshinori Takao, Koji Eriguchi, and Kouichi Ono

Citation: Journal of Vacuum Science \& Technology B 32, 031212 (2014); doi: 10.1116/1.4874309

View online: http://dx.doi.org/10.1116/1.4874309

View Table of Contents: http://scitation.aip.org/content/avs/journal/jvstb/32/3?ver=pdfcov

Published by the AVS: Science \& Technology of Materials, Interfaces, and Processing

\section{Articles you may be interested in}

Two modes of surface roughening during plasma etching of silicon: Role of ionized etch products

J. Appl. Phys. 116, 223302 (2014); 10.1063/1.4903956

Surface loss rates of $\mathrm{H}$ and $\mathrm{Cl}$ radicals in an inductively coupled plasma etcher derived from time-resolved electron density and optical emission measurements

J. Vac. Sci. Technol. A 28, 360 (2010); 10.1116/1.3330766

Investigation of surface modifications of 193 and $248 \mathrm{~nm}$ photoresist materials during low-pressure plasma etching

J. Vac. Sci. Technol. B 22, 2594 (2004); 10.1116/1.1805545

Modeling of fluorine-based high-density plasma etching of anisotropic silicon trenches with oxygen sidewall passivation

J. Appl. Phys. 94, 6311 (2003); 10.1063/1.1621713

Mechanisms for $\mathrm{CF} 2$ radical generation and loss on surfaces in fluorocarbon plasmas

J. Vac. Sci. Technol. A 18, 2661 (2000); 10.1116/1.1319816

\section{HIDEN}

\section{Instruments for Advanced Science}

w www.HidenAnalytical.com E info@hiden.co.uk

CLICK TO VIEW our product catalogue

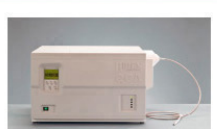

Gas Analysis

dynamic measurement of reaction gas streams

catalysis and thermal analysis

molecular beam studies

, ermetation enironmentat and ecological studes

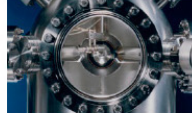

Surface Science

UHVTPD

SIMS elemental imaging - surface mapping

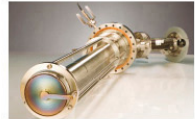

Plasma Diagnostics plasma source characterization etch and deposition process reaction , analysis of neutral and radical species

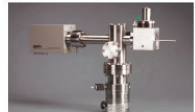

Vacuum Analysis , partial pressure measurement and contro of process gases reactive sputter process contro vacuum diagnostics Teum coatin pross monitoring 


\title{
Surface roughening and rippling during plasma etching of silicon: Numerical investigations and a comparison with experiments
}

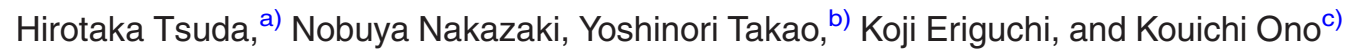 \\ Department of Aeronautics and Astronautics, Graduate School of Engineering, Kyoto University, \\ Kyoto-daigaku Katsura, Nishikyo-ku, Kyoto 615-8540, Japan
}

(Received 23 December 2013; accepted 21 April 2014; published 14 May 2014)

Atomic- or nanometer-scale surface roughening and rippling during $\mathrm{Si}$ etching in high-density $\mathrm{Cl}_{2}$ and $\mathrm{Cl}_{2} / \mathrm{O}_{2}$ plasmas have been investigated by developing a three-dimensional atomic-scale cellular model (ASCeM-3D), which is a 3D Monte Carlo-based simulation model for plasma-surface interactions and the feature profile evolution during plasma etching. The model took into account the behavior of $\mathrm{Cl}^{+}$ions, $\mathrm{Cl}$ and $\mathrm{O}$ neutrals, and etch products and byproducts of $\mathrm{SiCl}_{x}$ and $\mathrm{SiCl}_{x} \mathrm{O}_{y}$ in microstructures and on feature surfaces therein. The surface chemistry and kinetics included surface chlorination, chemical etching, ion-enhanced etching, sputtering, surface oxidation, redeposition of etch products desorbed from feature surfaces being etched, and deposition of etch byproducts coming from the plasma. The model also took into account the ion reflection or scattering from feature surfaces on incidence and/or the ion penetration into substrates, along with geometrical shadowing of the feature and surface reemission of neutrals. The simulation domain was taken to consist of small cubic cells of atomic size, and the evolving interfaces were represented by removing $\mathrm{Si}$ atoms from and/or allocating them to the cells concerned. Calculations were performed for square substrates $50 \mathrm{~nm}$ on a side by varying the ion incidence angle onto substrate surfaces, typically with an incoming ion energy, ion flux, and neutral reactant-to-ion flux ratio of $E_{i}=100 \mathrm{eV}, \Gamma_{i}^{0}=1.0 \times 10^{16} \mathrm{~cm}^{-2} \mathrm{~s}^{-1}$, and $\Gamma_{n}^{0} / \Gamma_{i}^{0}=100$. Numerical results showed that nanoscale roughened surface features evolve with time during etching, depending markedly on ion incidence angle; in effect, at $\theta_{i}=0^{\circ}$ or normal incidence, concavo-convex features are formed randomly on surfaces. On the other hand, at increased $\theta_{i}=45^{\circ}$ or oblique incidence, ripple structures with a wavelength of the order of $15 \mathrm{~nm}$ are formed on surfaces perpendicularly to the direction of ion incidence; in contrast, at further increased $\theta_{i} \geq 75^{\circ}$ or grazing incidence, small ripples or slitlike grooves with a wavelength of $<5 \mathrm{~nm}$ are formed on surfaces parallel to the direction of ion incidence. Such surface roughening and rippling in response to ion incidence angle were also found to depend significantly on ion energy and incoming fluxes of neutral reactants, oxygen, and etch byproducts. Two-dimensional power spectral density analysis of the roughened feature surfaces simulated was employed in some cases to further characterize the lateral as well as vertical extent of the roughness. The authors discuss possible mechanisms responsible for the formation and evolution of the surface roughness and ripples during plasma etching, including stochastic roughening, local micromasking, and effects of ion reflection, surface temperature, and ion angular distribution. Moreover, plasma etching experiments of blank $\mathrm{Si}$ substrates in $\mathrm{Cl}_{2}$ were conducted by varying the rf bias power or ion incident energy to verify the validity of our ASCeM-3D model. A comparison of the etch rate and root-mean-square (rms) surface roughness between experiments and simulations indicated that the ASCeM-3D with $\theta_{i}=0^{\circ}$ reproduces well the experiments at $E_{i}<250 \mathrm{eV}$, while does not reproduce the rms roughness at higher $E_{i}>250 \mathrm{eV}$, where the roughness decreases with increasing $E_{i}$ in experiments, while continues to increase with $E_{i}$ in simulations. Possible mechanisms for this disagreement at increased $E_{i}$ are discussed with the help of several plasma and surface diagnostics and classical molecular dynamics simulations for $\mathrm{Si} / \mathrm{Cl}$ and $\mathrm{Si} / \mathrm{SiCl}$ systems. (C) 2014 American Vacuum Society.

[http://dx.doi.org/10.1116/1.4874309]

\footnotetext{
${ }^{a}$ Present address: Center for Semiconductor Research \& Development, Toshiba Corporation Semiconductor \& Storage Products Company, Komukai Toshiba-cho, Saiwai-ku, Kawasaki 212-8583, Japan.

${ }^{b)}$ Present address: Division of Systems Research, Faculty of Engineering, Yokohama National University, Tokiwadai, Hodogaya-ku, Yokohama 240-8501, Japan.

${ }^{c}$ Electronic mail: ono@kuaero.kyoto-u.ac.jp
}

\section{INTRODUCTION}

Plasma etching technology is now widely employed in manufacturing integrated devices such as ultralarge-scale integrated (ULSI) circuit devices ${ }^{1,2}$ and microelectromehanical systems (MEMSs). ${ }^{3}$ As ULSI circuit device dimensions continue to be scaled down, ${ }^{4}$ increasingly strict requirements are being imposed on plasma etching technology. 5 The requirements include the precise control of profile, critical dimension (CD), and their microscopic uniformity (or 
aspect-ratio dependence), together with that of etch rate, selectivity, and damage. The precise control of Si etching in $\mathrm{Cl}$ - and Br-based plasmas is indispensable for the fabrication of gate electrodes and shallow trench isolation, ${ }^{1,2}$ through suppressing profile anomalies of sidewalls and bottom surfaces of the feature. Sidewall anomalies, such as tapering, bowing, footing (or bottom corner rounding), and notching, largely affect the CD and its difference between isolated and dense patterns and also between $p$ - and $n$-type gates. ${ }^{6}$ Anomalies of bottom surfaces, such as microtrenching and roughening, affect the uniformity of bottom surfaces and lead to pitting or punchthrough of gate oxides. ${ }^{7}$

Atomic- or nanometer-scale roughness on etched feature surfaces has also become an important issue to be resolved in the fabrication of nanoscale microelectronic devices, because the roughness formed during plasma etching ${ }^{8-31}$ would be comparable to the $\mathrm{CD}$ and the thickness of the layer being etched and/or the layer underlying. In gate fabrication, the roughness on feature sidewalls is responsible for the line edge roughness (LER) and line width roughness (LWR), ${ }^{32,33}$ which cause the variability in gate or channel length and thus lead to that in transistor performance. ${ }^{34,35}$ Moreover, in advanced three-dimensional (3D) device structures such as fin-type field effect transistors (FinFETs), ${ }^{5,34,36}$ the effects of the fin as well as the gate LER and LWR become significant, ${ }^{34}$ because LER and LWR occur also in the fin etch process, 5,36 and the conducting channel of FinFETs is formed on the top and sidewall surfaces of the fin. The roughness at the feature bottom affects the uniformity of bottom surfaces, which is responsible for a recess and damage in substrates, ${ }^{37}$ and, in turn, also leads to the variability in transistor performance.

The surface roughness formed during plasma etching of $\mathrm{Si}$ is also an important issue in MEMS fabrication processes, although the device dimensions are much larger than those of ULSI devices. In practice, the surface roughness is appreciated to affect the performance of MEMS through the fracture strength of fabricated microstructures ${ }^{38}$ and the friction force between moving microparts. ${ }^{39}$ Additionally, the processing time for etching deep microstructures is significantly long as compared to that in ULSI processes, which in turn would lead to the growth of more significant surface roughness in MEMS processes.

Several experiments have been concerned with the formation and evolution of surface roughness during etching of blank (or planar) $\mathrm{Si}$ wafers in $\mathrm{Cl}_{2},{ }^{8,11,25} \mathrm{SF}_{6}, 9,17,26,31$ $\mathrm{CF}_{4} / \mathrm{O}_{2},{ }^{14}$ and $\mathrm{Ar}$ (Ref. 24) plasmas, and also in $\mathrm{XeF}_{2}$ gases with $^{18}$ and without ${ }^{12} \mathrm{Ar}^{+}$ion beams. Correspondingly, several theoretical and/or numerical studies have been made to interpret the roughness experimentally observed in plasma etching, using a continuum model, ${ }^{13-16}$ Monte Carlo simulation, ${ }^{15,16,22,23,28,29,31}$ and classical molecular dynamics (MD) simulation. ${ }^{10,30}$ The low-level surface roughening at the initial stage of plasma exposure is generally assumed to be caused by the noise (or stochastic roughening due to the uniformity of incident fluxes of ions and/or neutral etchants on surfaces at microscale); ${ }^{13-16,24,28}$ however, it is not sufficient for interpreting the following evolution of roughness with time, which has further invoked a few qualitative mechanisms such as geometrical shadowing, ${ }^{13}$ surface reemission of etchants, ${ }^{14-16,22}$ and effects of etch inhibitors. ${ }^{23,31}$ More generic $(2+1)$-dimensional or 3D Monte Carlo simulations have recently been performed to reproduce the surface features roughened in $\mathrm{Ar}$ (Ref. 28) and $\mathrm{SF}_{6}$ (Ref. 31) plasma etching of $\mathrm{Si}$, taking into account surface chemistries such as sputtering and ion-enhanced etching with inhibitor deposition. However, the mechanisms for surface roughening during plasma etching are not yet fully understood, depending on a number of factors in processing.

Moreover, a number of experiments have been concerned with the surface roughness formed on feature sidewalls during plasma etching of patterned $\mathrm{Si}$ wafers to understand the origin of LER and LWR, and to find the way of reducing them as much as possible in the fabrication process for microelectronic devices. ${ }^{32-36}$ Sidewall roughening of the feature being etched is assumed to be caused by the pattern transfer of the mask edge roughness (resulting from lithography) into the underlayers being etched and also by that of grain boundaries of polycrystalline films to be etched into themselves. ${ }^{32-36}$ In practice, the addition of depositive gas species such as $\mathrm{CF}_{4}$ and/or reactive gases such as $\mathrm{HBr}$ giving depositive etch byproducts is often invoked to control and suppress the sidewall roughness in poly-Si gate and single-crystalline $\mathrm{Si}$ (c-Si) fin fabrication processes, ${ }^{32,36}$ through etch inhibitor deposition or passivation layer formation on feature sidewalls. On the other hand, the sidewall roughness would also be caused by plasma-surface interactions themselves on feature sidewalls, where the ion incidence is assumed to be off normal or oblique to the surface being etched. However, in plasma etching for pattern definition, it is hard to distinguish the sidewall roughness caused by plasma-surface interactions from that caused by especially the pattern transfer of the mask edge roughness. Only several studies of Sawin et al. have so far been concerned with the sidewall roughening originating from plasmasurface interactions during plasma etching, through investigations of the effects of the oblique ion incidence on blank substrates: 3D cellular Monte Carlo simulations of Si (Ref. 28) and $\mathrm{SiO}_{2}$ (Refs. 28 and 29) etching in $\mathrm{Ar}$ and $\mathrm{C}_{4} \mathrm{~F}_{8} / \mathrm{Ar}$ plasmas, respectively, and the experiments of Ar plasma beam etching of $\mathrm{Si}, \mathrm{SiO}_{2}$, and low dielectric constant (low-k) films, ${ }^{24} \mathrm{C}_{2} \mathrm{~F}_{6} /$ Ar plasma beam etching of low- $k$ films, ${ }^{20}$ and $\mathrm{C}_{4} \mathrm{~F}_{8} / \mathrm{Ar}$ plasma beam etching of $\mathrm{SiO}_{2}$ (Refs. 21 and 29) and low- $k$ films. ${ }^{21}$

We have developed a semi-empirical, atomic-scale cellular model (ASCeM) for simulating plasma-surface interactions and the resulting feature profile evolution during plasma etching of $\mathrm{Si}$, based on the Monte Carlo algorithm with the cell removal method for interface evolution; ${ }^{40-47}$ in effect, a two-dimensional ASCeM model (ASCeM-2D) reproduced the evolution of nanometer-scale profile anomalies on sidewalls and bottom surfaces of the feature, together with pattern-size or aspect-ratio dependences of them, during Si trench etching in $\mathrm{Cl}_{2}$ and $\mathrm{Cl}_{2} / \mathrm{O}_{2}$ plasmas. ${ }^{40-45}$ However, the ASCeM-2D was found to be insufficient to reproduce the nanoscale surface roughness formed during plasma etching, 
probably because the formation and evolution of roughness is $3 \mathrm{D}$ as well as stochastic. ${ }^{24,28}$ Therefore, we have recently developed a 3D ASCeM model (ASCeM-3D) to simulate the evolution of 3D feature profiles, including nanoscale surface features such as roughness, during $\mathrm{Si}$ etching in Cl-based plasmas. ${ }^{46,47}$ Preliminary results showed nanoscale surface roughening and also rippling during $\mathrm{Si}$ etching in $\mathrm{Cl}_{2}$ plasmas, in response to ion incidence angle onto blank substrate surfaces, ${ }^{47}$ as has been indicated numerically and experimentally in Ar plasma etching of $\mathrm{Si}^{24,28}$

This paper presents more details of the ASCeM-3D simulation of $\mathrm{Si}$ etching in $\mathrm{Cl}_{2}$ and $\mathrm{Cl}_{2} / \mathrm{O}_{2}$ plasmas, together with a comparison with our plasma etching experiments. Emphasis is placed on a better understanding of plasmasurface interactions that are responsible for the nanoscale surface roughening and rippling during plasma etching. It should be noted that on nanometer scale, stochastic mechanisms would be more important, because reactive particles incident on surfaces during etching are few in number; e.g., the number of incident ions is of the order of 100 , during etching $1-\mathrm{nm}^{2}$ surfaces in a depth of $1 \mathrm{~nm}$ (as will be mentioned later). Section II describes the ASCeM-3D model and numerical procedures. Numerical results are then given for the formation and evolution of surface roughness and ripples in response to ion incidence angle, including effects of plasma parameters such as ion incident energy, neutral reactant-to-ion flux ratio, and surface inhibitor-to-ion flux ratio. The etch or surface inhibitors concerned are oxygen and etch byproducts, and the roughened feature surfaces are analyzed in terms of vertical and in some cases lateral extent of the roughness. Then, Sec. III gives the experimental setup and the results for inductively coupled plasma (ICP) etching of $\mathrm{Si}$ in $\mathrm{Cl}_{2}$, together with those of several plasma and surface diagnostics. A comparison of the etch rate and root-meansquare (rms) surface roughness is made between the experiments and the ASCeM-3D simulations with normal ion incidence as a function of rf bias power or ion incident energy to verify the validity of our ASCeM-3D model. Some MD simulations are also invoked to further understand the atomistic mechanisms related to effects of etch byproduct ions. Finally, Sec. IV summarizes conclusions of this paper.

\section{NUMERICAL ANALYSIS}

\section{A. Model}

\section{Simulation domain}

Figure 1 shows a schematic of the ASCeM-3D model for Si etching in $\mathrm{Cl}_{2}$ and $\mathrm{Cl}_{2} / \mathrm{O}_{2}$ plasmas, together with the coordinate system $(x, y, z)$ presently employed. The ASCeM-3D methodology has been described in part in our previous papers, ${ }^{46,47}$ together with the surface chemistry and kinetics concerned, which is basically an extension of the ASCeM-2D. ${ }^{40-45}$ In more detail, the simulation domain is a square $W=50 \mathrm{~nm}$ on a side with a depth of $630 \mathrm{~nm}$, consisting of a number of small cubic cells of atomic size $L=\rho_{\mathrm{Si}}{ }^{-1 / 3}=2.7 \AA\left(185 \times 185 \times 2333 \approx 8 \times 10^{7}\right.$ cells in total), where $\rho_{\mathrm{Si}}=5.0 \times 10^{22} \mathrm{~cm}^{-3}$ is the atomic density of $\mathrm{Si}$ substrates. The substrates initially occupy a lower
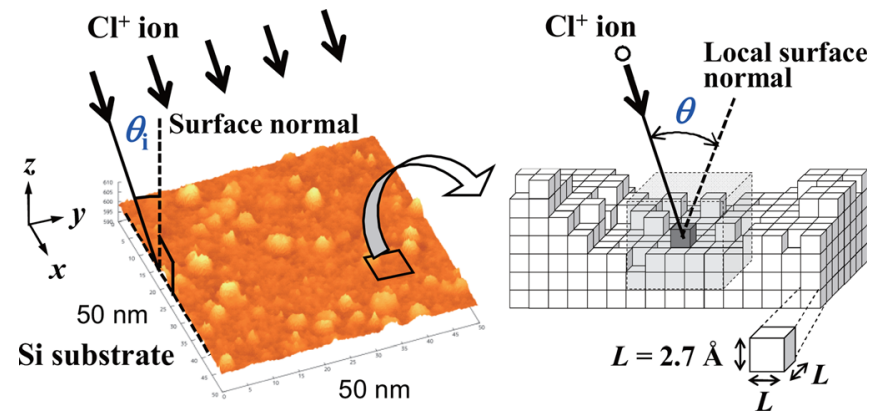

FIG. 1. (Color online) Schematic of our Monte Carlo-based ASCeM-3D model for simulating plasma-surface interactions and the feature profile evolution during plasma etching, together with the coordinate system $(x, y$, $z$ ) presently employed. The simulation domain is a square $50 \mathrm{~nm}$ on a side with an initial depth of $630 \mathrm{~nm}$, which is divided into a number of small cubic cells of atomic size $L=2.7 \AA$. In the figure, $\theta_{i}$ is the ion incidence angle onto substrate surfaces, while $\theta$ is the local ion incidence angle on microscopically roughened feature surfaces thereon. The local surface normal on feature surfaces is calculated by the extended four-point technique for $5 \times 5 \times 5$ neighboring cells $(125$ cells in total) at around the substrate surface cell that the ion reaches.

620-nm-deep layer therein (or the substrate surfaces are initially flat, being located $10 \mathrm{~nm}$ downward from the top of the domain). The cells are assumed to be rigid, and Si atoms are allocated at the center of substrate cells as a point mass. It should be noted that the atomic-scale cell size $L$ presently employed is one of the prominent features characterizing the ASCeM model, being about an order of magnitude smaller than that of other cell-based simulation models. ${ }^{22,23,28,29,31}$ In addition, the ASCeM cannot reproduce phenomena depending on the crystallinity of substrates, because it is not taken in the model.

\section{Particle injection and transport}

We consider energetic ions $\left(\mathrm{Cl}^{+}\right)$, neutral reactants or etchants $(\mathrm{Cl})$, reactive neutrals $(\mathrm{O})$, and etch and/or sputter byproducts $\left(\mathrm{SiCl}_{x}, \mathrm{SiCl}_{x} \mathrm{O}_{y}\right)$, which are randomly allocated at the top of the simulation domain, being successively injected therefrom (or from the plasma) onto substrates with given energies, fluxes, and angular distributions; ${ }^{42,46}$ concretely, the incoming ions are taken to have a Gaussian-like anisotropic angular distribution around the incidence angle $\theta_{i}$, while neutrals have an isotropic distribution. Moreover, etch and/or sputter products $\left(\mathrm{SiCl}_{x}, \mathrm{SiCl}_{x} \mathrm{O}_{y}\right)$ are taken to be desorbed from substrate surfaces being etched into vacuum, thermally or isotropically with the so-called cosine distribution. These incoming particles and desorbed products are also assumed to be point masses.

Transport of ions and neutrals in the ASCeM-3D is analyzed in three dimensions $(x, y, z)$ with periodical boundary conditions in the horizontal direction, based on singleparticle trajectories with three velocity components $\left(v_{x}, v_{y}\right.$, $\left.v_{z}\right)$. The particles are assumed to move straight from the top of the simulation domain onto substrate surfaces and then into microstructures thereon, without collisions with other particles in vacuum, where their transport is calculated every movement of step $L$, taking into account geometrical shadowing effects of the structure; then, the particles are assumed 
to reach the surface if there is a Si atom in any of the 26 cells neighboring the cell which the particle concerned is in. The local surface normal and thus the local angle $\theta$ of incidence on microstructural feature surfaces is calculated by using the extended four-point technique ${ }^{40,42,46,48}$ for $5 \times 5 \times 5$ neighboring cells ( 125 cells in total) at around the substrate surface cell that the particle reaches, as shown in Fig. 1; this is one of the key procedures in the cell-based simulations such as ASCeM, because the surface chemistry and kinetics calculations rely crucially on the local incidence angle $\theta$, as mentioned below. Note that $\theta=\theta_{i}$ for completely planar substrate surfaces. In addition, feature surfaces are taken to be charge neutral during etching, owing to the Auger process for energetic ions incident on surfaces.

\section{Surface chemistry and kinetics}

The ASCeM-3D takes into account surface chemistry and kinetics on substrates, ${ }^{42,46}$ based on the 3D Monte Carlo algorithm: surface chlorination (or adsorption and reemission of neutrals), chemical etching, ion-enhanced etching, sputtering, surface oxidation, redeposition of etch and/or sputter products desorbed from feature surfaces being etched, and deposition of etch and/or sputter byproducts coming from the plasma; the latter three lead to the formation of surface inhibitor or passivation layers, which are taken to be removed by sputtering through energetic ion bombardment. These are assumed to occur on substrate surface cells (or the outermost surface cells), each of which is allowed to contain four $\mathrm{Cl}$, two $\mathrm{Cl}$ and one $\mathrm{O}$, or two $\mathrm{O}$ atoms at maximum, in addition to one $\mathrm{Si}$ atom.

Surface chlorination is induced by adsorption of neutral reactants $\mathrm{Cl}$, where the sticking probability is taken to be $S_{n}=1-x / 4$ on $\operatorname{SiCl}_{x}(0 \leq x \leq 4)$ and $S_{n}=1-(x+2 y) / 4$ on $\mathrm{SiCl}_{x} \mathrm{O}_{y}(0 \leq x \leq 2$ for $y=1, x=0$ for $y=2)$ surfaces; otherwise, neutral $\mathrm{Cl}$ atoms are assumed to be reemitted thermally or reflected randomly with a probability $\left(1-S_{n}\right)$ from the surface into vacuum, which then move further toward another surfaces of the feature or go out of the simulation domain. Moreover, surface oxidation is induced by adsorption of strongly reactive $\mathrm{O}$ neutrals, where the sticking probability is taken to be $S_{o}=1$ on $\operatorname{SiCl}_{x}(0 \leq x \leq 4)$ and $S_{o}=1-y / 2$ on $\operatorname{SiCl}_{x} \mathrm{O}_{y}(0 \leq x \leq 2$ for $y=1, x=0$ for $y=2)$ surfaces, resulting in emission of displaced $\mathrm{Cl}$ atoms thermally or isotropically therefrom into vacuum; otherwise, neutral $\mathrm{O}$ atoms are also assumed to be reemitted thermally with a probability $\left(1-S_{o}\right)$, similarly as for $\mathrm{Cl}$ neutrals. Spontaneous or purely chemical etching is assumed to occur on fully chlorinated $\mathrm{SiCl}_{4}$ surfaces, and the corresponding reaction probability $\alpha_{\mathrm{SiCl} 4}$ is taken from the known data depending on surface temperature $T_{\mathrm{s}}$. ${ }^{49}$

The yield for ion-enhanced etching through energetic $\mathrm{Cl}^{+}$ ion incidence on chlorinated surfaces of $\mathrm{Si}$ is taken to be $Y_{\mathrm{SiCl} x}=(x / 4) Y_{\mathrm{SiCl} 4}(1 \leq x \leq 4)$, where $Y_{\mathrm{SiCl} 4}$ is the etch yield for $\mathrm{Cl}^{+}$on $\mathrm{Si}$ surfaces chlorine saturated. The yield $Y_{\mathrm{SiCl} 4}$ is taken from the known model depending on ion incident energy $E_{i}$ and angle $\theta$ on the surface, ${ }^{50}$ together with the sputter yield $Y_{\mathrm{Si}}$ for $\mathrm{Cl}^{+}$on $\mathrm{Si}^{51}$ then, the threshold energy is assumed to be $E_{\mathrm{th}}=12.0 \mathrm{eV}$ for $Y_{\mathrm{SiCl} 4},{ }^{52}$ and $E_{\mathrm{th}}=29.7 \mathrm{eV}$ for $Y_{\mathrm{Si}},{ }^{53}$ in view of the experiments of $\mathrm{Cl}_{2}$ plasma beam, ${ }^{52}$ $\mathrm{Cl}^{+}$plus $\mathrm{Cl} / \mathrm{Cl}_{2}$ beam, ${ }^{54}$ and $\mathrm{Cl}^{+}$beam etching of $\mathrm{Si}$ (Refs. 54 and 55) as well as the analytical models for sputtering. ${ }^{53}$ The etch or sputter yield for $\mathrm{Cl}^{+}$ions incident on oxidized $\mathrm{Si}$ surfaces is assumed to be $Y_{\mathrm{SiCl} x \mathrm{O} y}=4.0[(1+x+y) / 4]^{2} Y_{\mathrm{SiO} 2}$ $(0 \leq x \leq 2$ for $y=1)$, in view of the experiments of $\mathrm{Ar}^{+}$plus $\mathrm{Cl} / \mathrm{Cl}_{2}$ beam etching of $\mathrm{Si}$ and $\mathrm{SiO}_{2},{ }^{56}$ where $Y_{\mathrm{SiO} 2}$ is the sputter yield for $\mathrm{Cl}^{+}$on $\mathrm{SiO}_{2}$. The $E_{i}$ and $\theta$ dependences of $Y_{\mathrm{SiO} 2}$ are taken to be the same as those of $Y_{\mathrm{Si}}$, with the threshold $E_{\mathrm{th}}=40 \mathrm{eV} ;^{56}$ then, the etch selectivity is assumed to be $Y_{\mathrm{Si}} / Y_{\mathrm{SiO} 2}=8$ at $E_{i}=100 \mathrm{eV}$ and $\theta=0^{\circ}$, in view of the experiments of $\mathrm{Ar}^{+}$beam incidence on $\mathrm{Si}$ and $\mathrm{SiO}_{2}$ in $\mathrm{Cl}_{2} .{ }^{57}$

Redeposition of etch/sputter products desorbed from feature surfaces being etched, together with deposition of etch/ sputter byproducts coming from the plasma, is taken to occur according to the ballistic deposition model. ${ }^{58}$ The etch/sputter product and byproduct species concerned are silicon chlorides $\mathrm{SiCl}_{x}$, oxides $\mathrm{SiO}_{y}$, and oxychlorides $\mathrm{SiCl}_{x} \mathrm{O}_{y}$, in addition to $\mathrm{Si}$. The etch/sputter products are assumed to stick or redeposit on all feature surfaces (blank, chlorinated, oxidized, and deposited surfaces) with a probability $S_{q}=0.05 ;{ }^{42}$ otherwise, they are reemitted thermally or reflected randomly with a probability $\left(1-S_{q}\right)$ from the surface into vacuum, which then move further toward another surfaces of the feature or go out of the simulation domain. Similarly, the incoming etch/sputter byproducts are also assumed to stick or deposit on all feature surfaces with a probability $S_{p}=0.05,{ }^{42}$ and otherwise, they are reemitted thermally with a probability $\left(1-S_{p}\right)$. The removal of deposited surfaces is taken to be caused by etching and/or sputtering through energetic $\mathrm{Cl}^{+}$ion bombardment, according to the same chemistry and kinetics as mentioned above; then, the deposited $\mathrm{Si}$, $\mathrm{SiCl}_{x}, \mathrm{SiO}_{y}$, and $\mathrm{SiCl}_{x} \mathrm{O}_{y}$ are assumed to be removed or desorbed from the surface, thermally or isotropically with the cosine law, similarly as for the desorption of etch/sputter products and the surface reemission or reflection of neutrals.

Moreover, the ASCeM-3D takes into account two-body elastic collisions between energetic ions and substrate $\mathrm{Si}$ atoms, ${ }^{41,47}$ to analyze the ion reflection or scattering from feature surfaces on incidence into vacuum and the ion penetration into substrates. ${ }^{59,60}$ The analysis is also based on the 3D Monte Carlo calculation of ion trajectories according to the momentum and energy conservation through successive collisions with substrate $\mathrm{Si}$ atoms; then, the ions reflected from the surface into vacuum move further toward another surfaces of the feature or go out of the simulation domain. The interaction potential for $\mathrm{Cl}-\mathrm{Si}$ employed is the Ziegler-Biersack-Littmark (ZBL) type. ${ }^{61,62}$ In calculating the ion-related yields $Y_{\mathrm{Si}}, Y_{\mathrm{SiCl} x}, Y_{\mathrm{SiO} y}$, and $Y_{\mathrm{SiCl} x \mathrm{O} y}$, the ion energy concerned is taken to be its incident energy $E_{i}$ in the case of penetration, while the energy loss or deposit energy on surfaces $\Delta E_{i}=E_{i}-E_{r}$ in the case of reflection, where $E_{r}$ is the ion energy after reflection. It should be noted that the reflection and penetration of energetic ions incident on feature surfaces is a second one of the prominent features characterizing the ASCeM model; in practice, without the effects of ion reflection from surfaces on incidence, the ASCeM 
does not reproduce the evolution of 3D nanoscale surface features such as roughness and ripples as shown below and that of $2 \mathrm{D}$ nanoscale profile anomalies such as microtrench and micropillars as reported previously. ${ }^{43-45}$

\section{Surface advancement}

Surface advancement, or the evolution of interfaces between vacuum and substrate surfaces, is given by removal and/or addition of $\mathrm{Si}$ atoms to the cells concerned; ${ }^{42,46}$ in practice, $\mathrm{Si}$ atoms are removed from the substrate surface cells, together with the neutrals contained therein (or the outermost surface cells become empty), when the etching/ sputtering causes the desorption of etch/sputter products. On the other hand, $\mathrm{Si}$ atoms are newly allocated to the cells neighboring the outermost surface cells, together with the neutrals accompanied, when the redeposition and/or deposition of etch/sputter products occur. In addition, Sicontaining cells floating in vacuum often occur during simulation, which are removed by percolation according to the Hoshen-Kopelman algorithm; ${ }^{63-65}$ this is a second one of the key procedures in the cell-based simulations such as ASCeM, because unphysically roughened surface features tend to occur without percolation.

\section{B. Numerical results and discussion}

We simulate the evolution of surface features during $120 \mathrm{~s}$ after the start of etching, for different ion incidence angles of $\theta_{i}=0^{\circ}-85^{\circ}$, ion incident energies of $E_{i}=20-500 \mathrm{eV}$, incoming neutral reactant-to-ion flux ratios of $\Gamma_{n}^{0} / \Gamma_{i}^{0}=10-500$, incoming oxygen-to-ion flux ratios of $\Gamma_{o}^{0} / \Gamma_{i}^{0}=0-0.5$, and returning probabilities of $P_{r}=0-0.5$ for the etch/sputter products desorbed. Here, $P_{r}$ is the probability assumed originally in the ASCeM model, ${ }^{42}$ for the return of the etch/sputter products that have been desorbed from feature surfaces into vacuum and then gone out of the simulation domain (or gone out into the plasma) during etching; in practice, the incoming flux of etch/sputter byproducts from the plasma onto substrate surfaces is given by $\Gamma_{p}{ }^{0}=P_{r} \Gamma_{q}^{s}$ during etching, where $\Gamma_{q}^{s}=\rho_{\mathrm{Si}} \mathrm{ER}$ is the flux of etch/sputter products desorbed from feature surfaces being etched with an etch rate ER. An incoming ion flux $\Gamma_{i}^{0}=1.0 \times 10^{16} \mathrm{~cm}^{-2} \mathrm{~s}^{-1}$, ion temperature $k T_{i}=0.5 \mathrm{eV}\left(R=E_{i} / k T_{i}\right.$ is a measure of the angular distribution of incoming ion fluxes), and neutral or gas temperature $T_{g}=500 \mathrm{~K}$ are fixed in this study, together with the dopant concentration $N_{e}=1.0 \times 10^{18} \mathrm{~cm}^{-3}$ and surface temperature $T_{s}=320 \mathrm{~K}$ of substrates. These conditions are typical for $\mathrm{Si}$ etching in high-density plasmas, such as ICP and electron cyclotron resonance plasmas, ${ }^{66-68}$ where the ion-enhanced reactions are assumed to be dominant mechanisms for etching.

\section{Effects of ion incidence angle $\left(\theta_{i}\right)$}

Figure 2 shows typical snapshots of the surface features of $\mathrm{Si}$ (or typical 3D plots of the location of the outermost substrate cells on etched surfaces) at $t=20 \mathrm{~s}$ after the start of etching in $\mathrm{Cl}_{2}$ plasma, simulated for different ion incidence angles $\theta_{i}=0^{\circ}, 45^{\circ}, 75^{\circ}$, and $80^{\circ}$. Calculations were made with an ion energy $E_{i}=100 \mathrm{eV}$ and neutral-to-ion flux ratio
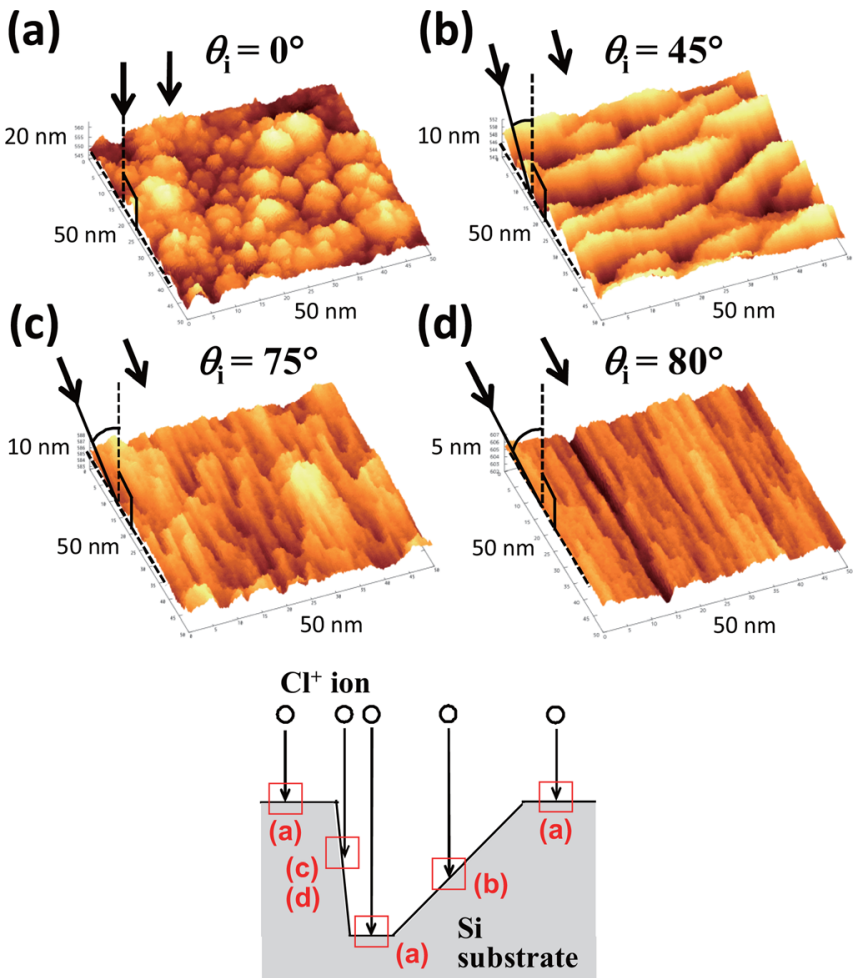

FIG. 2. (Color online) Typical surface features of Si at $t=20 \mathrm{~s}$ after the start of etching in $\mathrm{Cl}_{2}$ plasma, simulated with the ASCeM-3D for different ion incidence angles of $\theta_{i}=$ (a) $0^{\circ}$, (b) $45^{\circ}$, (c) $75^{\circ}$, and (d) $80^{\circ}$. Inset is a schematic of the corresponding situations on patterned feature surfaces. Calculations were made with an ion energy $E_{i}=100 \mathrm{eV}$, ion temperature $k T_{i}=0.5 \mathrm{eV}$, ion flux $\Gamma_{i}^{0}=1.0 \times 10^{16} \mathrm{~cm}^{-2} \mathrm{~s}^{-1}$, and neutral-to-ion flux ratio $\Gamma_{n}^{0} / \Gamma_{i}^{0}=100$, in the absence of incoming fluxes of oxygen and etch/sputter byproducts from the plasma $\left(\Gamma_{o}{ }^{0}=\Gamma_{p}{ }^{0}=0\right)$. The neutral or gas temperature was taken to be $T_{g}=500 \mathrm{~K}$, the surface temperature of substrates $T_{s}=320 \mathrm{~K}$, and the dopant concentration therein $N_{e}=1.0 \times 10^{18} \mathrm{~cm}^{-3}$.

$\Gamma_{n}^{0} / \Gamma_{i}^{0}=100$ in the absence of surface oxidation and deposition of etch/sputter byproducts $\left(\Gamma_{o}^{0}=\Gamma_{p}{ }^{0}=0\right)$. Note that the angle $\theta_{i}=0^{\circ}$ corresponds to the situation on top and bottom surfaces of patterned features being etched, and the increased $\theta_{i}=45^{\circ}, 75^{\circ}$, and $80^{\circ}$ to the situation on tapered sidewalls of the feature, as inset schematically in the figure. The results indicate that roughened features of the order of a few $\mathrm{nm}$ occur on etched surfaces, depending markedly on the angle $\theta_{i}$ of ion incidence. In effect, at $\theta_{i}=0^{\circ}$ or normal incidence, concavo-convex features are formed randomly on surfaces. On the other hand, at increased $\theta_{i}=45^{\circ}$ or oblique incidence, ripple structures are formed on surfaces perpendicularly to the direction of ion incidence; in contrast, at further increased $\theta_{i}=75^{\circ}$ and $80^{\circ}$ or grazing incidence, small ripples or slitlike grooves are formed on surfaces parallel to the direction of ion incidence. Similar surface features were found to occur in response to $\theta_{i}$, for rectangular substrates of $50 \times 100 \mathrm{~nm}^{2}$ and $100 \times 50 \mathrm{~nm}^{2}$ as well as for square ones of $50 \times 50 \mathrm{~nm}^{2}$.

Figure 3 shows the time evolution of surface features of Si during etching in $\mathrm{Cl}_{2}$ plasma, simulated for different ion incidence angles in the range $\theta_{i}=0^{\circ}-80^{\circ}$ under similar calculation conditions of Fig. $2\left(E_{i}=100 \mathrm{eV}, \Gamma_{n}{ }^{0} / \Gamma_{i}^{0}=100\right.$, $\left.\Gamma_{o}{ }^{0}=\Gamma_{p}{ }^{0}=0\right)$. The results indicate that soon after the start 


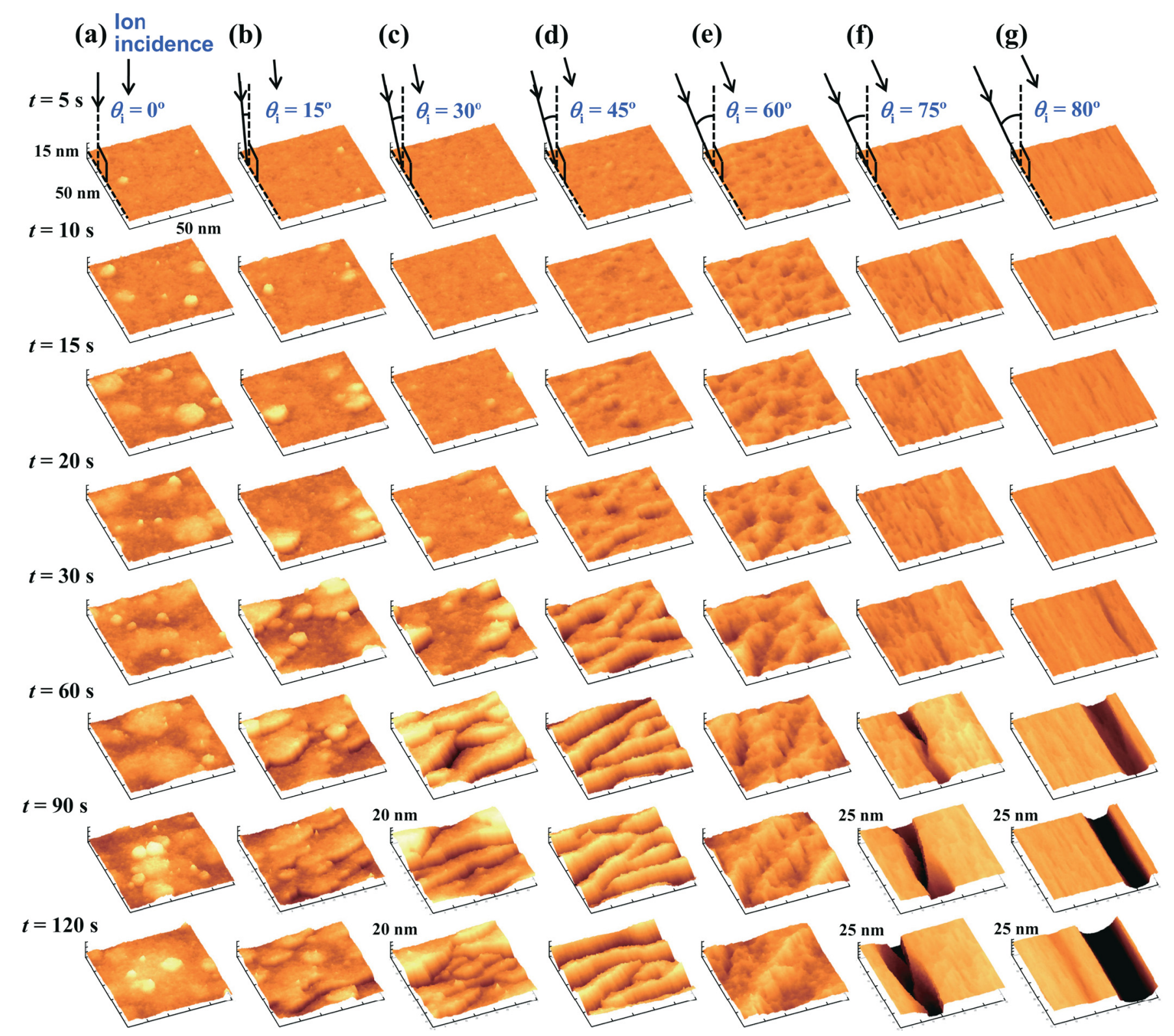

FIG. 3. (Color online) Time evolution of the surface features of $\mathrm{Si}$ etched in $\mathrm{Cl}_{2}$ plasma, simulated with the ASCeM-3D for different ion incidence angles of $\theta_{i}=\left(\right.$ a) $0^{\circ}$, (b) $15^{\circ}$, (c) $30^{\circ}$, (d) $45^{\circ}$, (e) $60^{\circ}$, (f) $75^{\circ}$, and (g) $80^{\circ}$ under similar calculation conditions of Fig. $2\left(E_{i}=100 \mathrm{eV}, \Gamma_{n}^{0} / \Gamma_{i}^{0}=100, \Gamma_{o}^{0}=\Gamma_{p}^{0}=0\right)$. [Reproduced in part with permission from H. Tsuda, Y. Takao, K. Eriguchi, and K. Ono, Jpn. J. Appl. Phys. 51, 08 HC01 (2012). Copyright 2012 The Japan Society of Applied Physics.]

of etching, the initially planar surfaces are roughened randomly with small concavo-convex features at any angle $\theta_{i}$ of ion incidence, while the evolution of surface features thereafter depends largely on $\theta_{i}$. At $\theta_{i}=0^{\circ}$ or normal incidence, small concavo-convex features continue to evolve into larger ones with time. On the other hand, at $\theta_{i}=45^{\circ}$ or oblique incidence, small concavo-convex features tend to merge with each other with time $(t>5 \mathrm{~s})$ to form small convex features elongated perpendicularly to the direction of ion incidence, which evolve further into apparent ripple structures perpendicular to the direction of ion incidence. The wavelength and amplitude (peak-to-peak) of the ripple are about 13 and $6.5 \mathrm{~nm}$, respectively, traveling with a speed of about $5 \mathrm{~nm} / \mathrm{s}$ (slightly higher than the etch rate) in the direction of ion incidence. At intermediate $\theta_{i}=15^{\circ}$ and $30^{\circ}$, the surface features exhibit an intermediate appearance, or a transition from randomly to regularly roughened surface features with increasing $\theta_{i}$.

At $\theta_{i}=75^{\circ}$ and $80^{\circ}$ or grazing incidence, small grooves elongated parallel to the direction of ion incidence occur at relatively early times $(t<5 \mathrm{~s})$ after the start of etching, which evolve further into small ripples or slitlike grooves parallel to the direction of ion incidence. The wavelength and amplitude of the ripple are both $<5 \mathrm{~nm}$. It is noted here that the slitlike grooves are longer at $\theta_{i}=80^{\circ}$ than at $75^{\circ}$, and that at $\theta_{i} \geq 75^{\circ}$, some of them tend to enlarge significantly with time to form a wide, deep groove $(>10 \mathrm{~nm}$ in width and $>30 \mathrm{~nm}$ in depth) on etched surfaces; in the simulation, such wide, deep grooves appear at some times and do not appear at other times, which is attributable presumably to the Monte Carlo calculation of stochastic processes using random numbers, and may occur on tapered sidewalls during 
plasma etching for pattern definition. At an intermediate $\theta_{i}=60^{\circ}$, the surface features exhibit an intermediate appearance or a transition of the ripple structures from perpendicular to parallel to the direction of ion incidence with increasing $\theta_{i}$.

Figure 4 shows the etch rate and rms surface roughness as a function of etching time $t$ for different ion incidence angles $\theta_{i}$ derived from the simulations of Fig. 3. Here, the rms roughness at time $t$ is calculated from the rms average or standard deviation of the surface feature ordinates from their mean value

$$
\mathrm{RMS}=\sqrt{\frac{1}{N^{2}} \sum_{m=1}^{N} \sum_{n=1}^{N}[z(m, n)-\bar{z}]^{2}}
$$

where $(m, n)$ denotes the location of the outermost substrate cells in the $(x, y)$ coordinates, $z(m, n)$ denotes the ordinate at the cell location $(m, n)$ at time $t, \bar{z}=\left(1 / N^{2}\right) \sum_{m=1}^{N} \sum_{n=1}^{N}$ $z(m, n)$ is the arithmetic average of the $z$ values within the surface area of $W^{2}=50 \times 50 \mathrm{~nm}^{2}$ concerned, and $N^{2}=185$ $\times 185$ is the number of the cell locations or data points therein. Moreover, the etch rate at time $t$ is also calculated from the difference in the average or mean ordinate $\bar{z}$ between $t+\Delta t$ and $t: \mathrm{ER}=[\bar{z}(t+\Delta t)-\bar{z}(t)] / \Delta t$. The etch rate at any angle $\theta_{i}$ of incidence exhibits a significant increase immediately after the start of etching and then maintains an almost constant value during etching. The etch rate is maximum at $\theta_{i}=0^{\circ}$ or normal incidence, decreasing with increasing $\theta_{i}$, which reflects the known angle
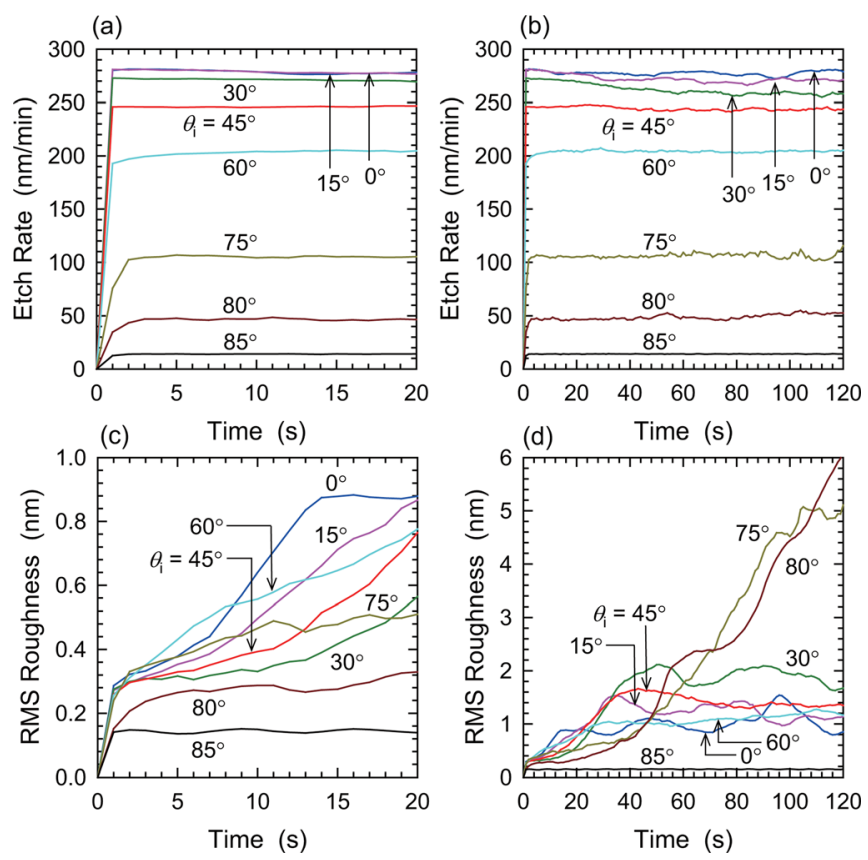

FIG. 4. (Color online) Etch rate as a function of etching time in the range (a) $0 \leq t \leq 20 \mathrm{~s}$ and (b) $0 \leq t \leq 120 \mathrm{~s}$, together with the rms surface roughness as a function of time in the range (c) $0 \leq t \leq 20 \mathrm{~s}$ and (d) $0 \leq t \leq 120 \mathrm{~s}$, for different ion incidence angles in the range $0^{\circ} \leq \theta_{i} \leq 85^{\circ}$. These are the data derived from the simulations of Fig. 3. [Reproduced in part with permission from H. Tsuda, Y. Takao, K. Eriguchi, and K. Ono, Jpn. J. Appl. Phys. 51, 08HC01 (2012). Copyright 2012 The Japan Society of Applied Physics.] dependence of the etch yield taken in the ASCeM model for ion-enhanced etching reactions. ${ }^{50}$

In contrast, the rms surface roughness exhibits a markedly different behavior: the roughness increases gradually with time at any angle $\theta_{i}$ of incidence, after some induction period $(\mathrm{RMS} \approx 0.2-0.3 \mathrm{~nm}$ at $t<5 \mathrm{~s}$ ); then, the roughness tends to reach steady state (RMS $\approx 1-2 \mathrm{~nm}$ ) roughly in $t<60 \mathrm{~s}$ at $\theta_{i}=0^{\circ}-60^{\circ}$. On the other hand, at $\theta_{i}=75^{\circ}$ and $80^{\circ}$ or grazing incidence, the rms roughness continues to increase significantly with time (RMS $>5-6 \mathrm{~nm}$ at $t>120 \mathrm{~s}$ ), corresponding to the formation of a wide, deep slitlike groove as seen in Figs. 3(f) and 3(g). At further increased $\theta_{i} \geq 85^{\circ}$, the roughness remains small (RMS $\approx 0.2 \mathrm{~nm}$ ), without the following evolution with time. It is noted here that the evolution of the rms roughness at $\theta_{i}=0^{\circ}$ is not consistent with the results of plasma etching experiments of blank $\mathrm{Si}$ substrates in $\mathrm{SF}_{6}$ (Refs. 9, 23, 26, and 31) and $\mathrm{CF}_{4} / \mathrm{O}_{2}$ (Ref. 14) previously reported, which showed a continuous increase in roughness with time $>10-20 \mathrm{~min}$.

Nanoscale ripple structures formed on Si by off-normal or oblique ion incidence has attracted much attention in recent noble-gas ion-beam experiments $\left(\mathrm{Ne}^{+}, \mathrm{Ar}^{+}, \mathrm{Kr}^{+}\right.$, and $\mathrm{Xe}^{+}$ beams of several hundred $\mathrm{eV}$ to a few tens of $\mathrm{keV}),{ }^{69-76}$ from the viewpoint of the fabrication of well-organized nanostructures. A transition from randomly roughed to regularly rippled surface features and a transition of the ripples from perpendicular to parallel to the direction of ion incidence, which occur depending on beam incidence angle, have also attracted attention of theoretical studies, ${ }^{69,72,75,77-83}$ from the viewpoint of a fundamental interest in beam-surface interactions. In plasma environments, the oblique incidence of ions should occur on feature sidewalls during etching for pattern definition, and so the formation mechanisms for such surface ripples would be responsible for the LER and LWR formed thereon. However, it is difficult to carry out experiments to elucidate the effects of oblique ion incidence in plasma etching environments; in effect, the feature profiles are too small and/or too complex for nanoscale devices to diagnose plasma-surface interactions on patterned feature surfaces during etching. Moreover, it is hard to distinguish the sidewall roughness and/or ripples caused by plasma-surface interactions themselves from those caused by the other effects such as mask edge roughness, as mentioned earlier. The experiments using blank substrates are also difficult, because the ion incidence is usually normal to planar substrate surfaces after being accelerated through the sheath on substrates immersed in plasma. It is noted that in plasma environments, only a few numerical and experimental studies of Sawin et al. have so far been concerned with effects of the oblique ion incidence on blank $\mathrm{Si}$ substrates in $\mathrm{Ar}$ plasma beam etching, showing the nanoscale surface roughening and rippling in response to ion incidence angle onto surfaces, ${ }^{24,28}$ prior to our numerical study.

\section{Effects of ion energy $\left(E_{i}\right)$}

Figure 5 shows the surface features of $\mathrm{Si}$ at $t=60 \mathrm{~s}$ after the start of etching in $\mathrm{Cl}_{2}$ plasma, simulated for different ion incident energies in the range $E_{i}=20-500 \mathrm{eV}$ with incidence 

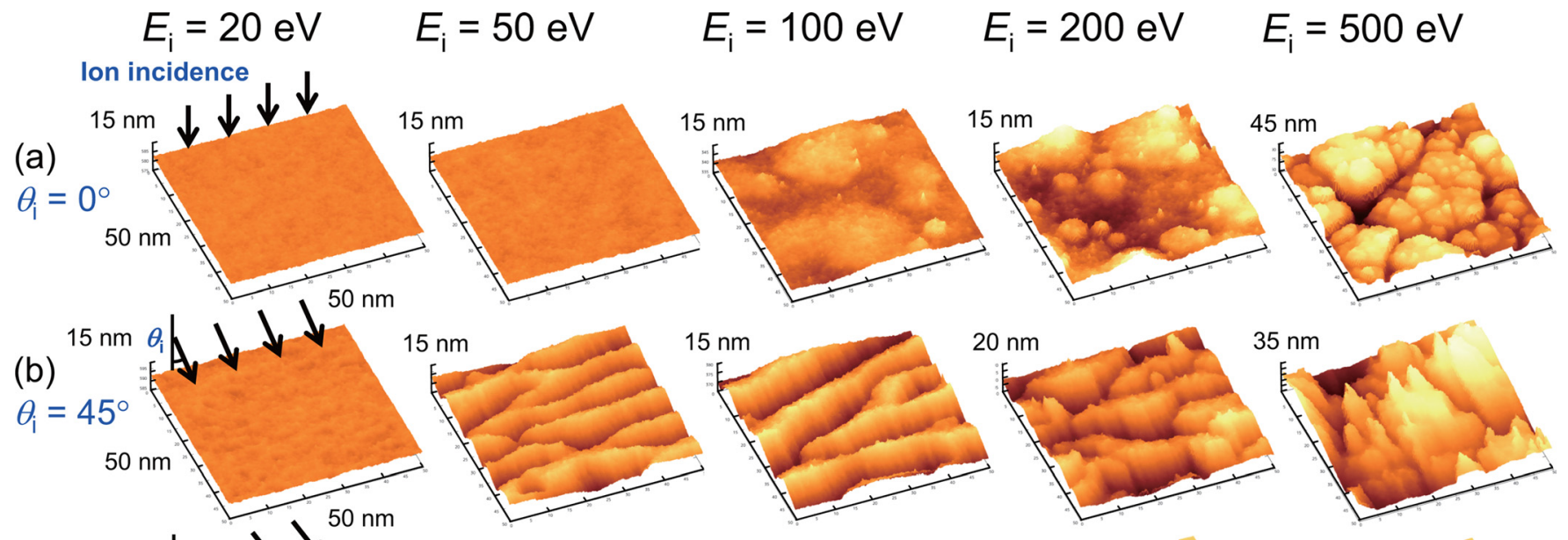

(c)
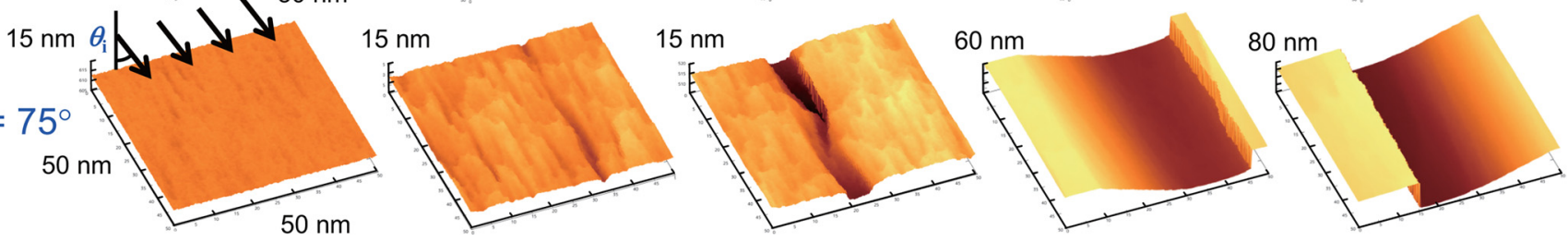

FIG. 5. (Color online) Surface features of $\mathrm{Si}$ at $t=60 \mathrm{~s}$ after the start of etching in $\mathrm{Cl}_{2}$ plasma, simulated for different ion energies in the range $E_{i}=20-500 \mathrm{eV}$ with ion incidence angles of $\theta_{i}=$ (a) $0^{\circ}$, (b) $45^{\circ}$, and (c) $75^{\circ}$. The other calculation conditions in the ASCeM-3D are the same as those of Fig. 3 ( $\Gamma_{n}{ }^{0} / \Gamma_{i}^{0}=100$, $\left.\Gamma_{o}{ }^{0}=\Gamma_{p}{ }^{0}=0\right)$. Note that the surface features for $E_{i}=100 \mathrm{eV}$ of interest here are the same as those shown at $t=60 \mathrm{~s}$ in Figs. 3(a), 3(d), and 3(f).

angles of $\theta_{i}=0^{\circ}, 45^{\circ}$, and $75^{\circ}$. The other calculation conditions in the ASCeM-3D are the same as those of Fig. 3 $\left(\Gamma_{n}^{0} / \Gamma_{i}^{0}=100, \Gamma_{o}^{0}=\Gamma_{p}^{0}=0\right)$. Note that the surface features for $E_{i}=100 \mathrm{eV}$ of interest here are the same as those shown at $t=60 \mathrm{~s}$ in Figs. 3(a), 3(d), and 3(f), and the corresponding etch rate and rms surface roughness are shown in Fig. 6 as a function of $E_{i}$. The etch rate increases with increasing ion energy $E_{i}$ at any incidence angle $\theta_{i}$; moreover, for a given $E_{i}$, the etch rate is larger at $\theta_{i}=0^{\circ}$ and $45^{\circ}$ than at $\theta_{i}=75^{\circ}$. These reflect the ion-enhanced etching characteristics of $\mathrm{Si}$ taken in the model or the known energy and angle dependences of the etch yield for ion-enhanced reactions. ${ }^{50,52,54}$

The rms roughness increases with increasing $E_{i}$ at $\theta_{i}=0^{\circ}$ and $45^{\circ}$. It is noted here that at $\theta_{i}=0^{\circ}$, the increase in roughness with $E_{i}$ is consistent with the results of some plasma etching experiments of blank $\mathrm{Si}$ substrates in $\mathrm{Cl}_{2}$ previously reported ${ }^{11}$ while it is inconsistent with those of some other plasma experiments of $\mathrm{Si}$ etching in $\mathrm{Cl}_{2}$ (Ref. 25) and
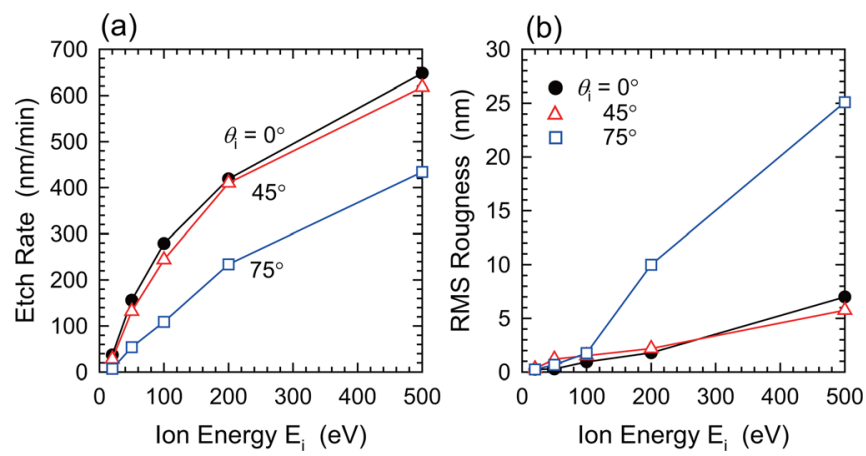

FIG. 6. (Color online) (a) Etch rate and (b) rms surface roughness at $t=60 \mathrm{~s}$ as a function of ion energy in the range $E_{i}=20-500 \mathrm{eV}$ with ion incidence angles of $\theta_{i}=0^{\circ}, 45^{\circ}$, and $75^{\circ}$ derived from the simulations of Fig. 5 .
$\mathrm{SF}_{6} ;{ }^{9,26}$ in effect, the experiments showed an increase ${ }^{11}$ and a decrease $e^{9,25,26}$ in roughness with increasing rf bias power or dc self-bias voltage. In Fig. 5(b), the ripple structure at $\theta_{i}=45^{\circ}$ is seen to be significant in the range $E_{i} \approx 50-200$ $\mathrm{eV}$, while it tends to fade away for further increased $E_{i}$ with the appearance of squamous features; in addition, the ripple wavelength at $\theta_{i}=45^{\circ}$ is about $13 \mathrm{~nm}$ for $E_{i}=100 \mathrm{eV}$, increasing with increasing $E_{i}$, which is consistent with the extrapolation of the experimental observations of $\mathrm{Ar}^{+}$and $\mathrm{Kr}^{+}$beam bombardment $\left(E_{i}=500-2000 \mathrm{eV}, \theta_{i}=15^{\circ}\right)$ on $\mathrm{c}-\mathrm{Si}^{71}$ On the other hand, the rms roughness at $\theta_{i}=75^{\circ}$ increases gradually and then significantly with increasing $E_{i}$; correspondingly, in Fig. 5(c), the ripple at $\theta_{i}=75^{\circ}$ is seen to enlarge significantly for increased $E_{i}>100 \mathrm{eV}$, resulting in a wide, deep groove.

\section{Effects of neutral flux $\left(\Gamma_{n}{ }^{0} \Gamma_{i}{ }^{0}\right)$}

Figure 7 shows the surface features of Si at $t=60 \mathrm{~s}$ after the start of etching in $\mathrm{Cl}_{2}$ plasma, simulated for different incoming neutral-to-ion flux ratios in the range $\Gamma_{n}^{0} / \Gamma_{i}^{0}=10-500$ with ion incidence angles of $\theta_{i}=0^{\circ}, 45^{\circ}$, and $75^{\circ}$. The other calculation conditions in the ASCeM-3D are the same as those of Fig. $3\left(E_{i}=100 \mathrm{eV}, \Gamma_{o}{ }^{0}=\Gamma_{p}{ }^{0}=0\right)$. Note that the surface features for $\Gamma_{n}^{0} / \Gamma_{i}^{0}=100$ of interest here are the same as those shown at $t=60 \mathrm{~s}$ in Figs. 3(a), $3(\mathrm{~d})$, and 3(f), and the corresponding etch rate and rms surface roughness are shown in Fig. 8 as a function of $\Gamma_{n}^{0} / \Gamma_{i}^{0}$. The etch rate increases and tends to be saturated with increasing neutral flux $\Gamma_{n}{ }^{0} / \Gamma_{i}^{0}$ at any incidence angle $\theta_{i}$; moreover, for a given $\Gamma_{n}^{0} / \Gamma_{i}^{0}$, the etch rate is larger at $\theta_{i}=0^{\circ}$ and $45^{\circ}$ than at $\theta_{i}=75^{\circ}$. These also reflect the ion-enhanced etching characteristics of $\mathrm{Si}$ taken in the 


$$
\Gamma_{n}{ }^{0} / \Gamma_{i}{ }^{0}=10 \quad \Gamma_{n} 0 / \Gamma_{i}{ }^{0}=50 \quad \Gamma_{n} / \Gamma_{i}{ }^{0}=100 \quad \Gamma_{n} 0 / \Gamma_{i}^{0}=200 \quad \Gamma_{n} 0 / \Gamma_{i}^{0}=500
$$

(a)
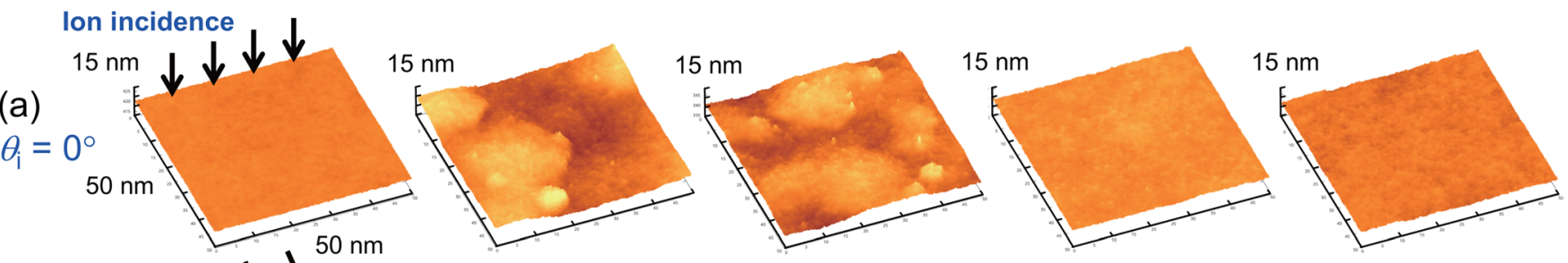

(b)
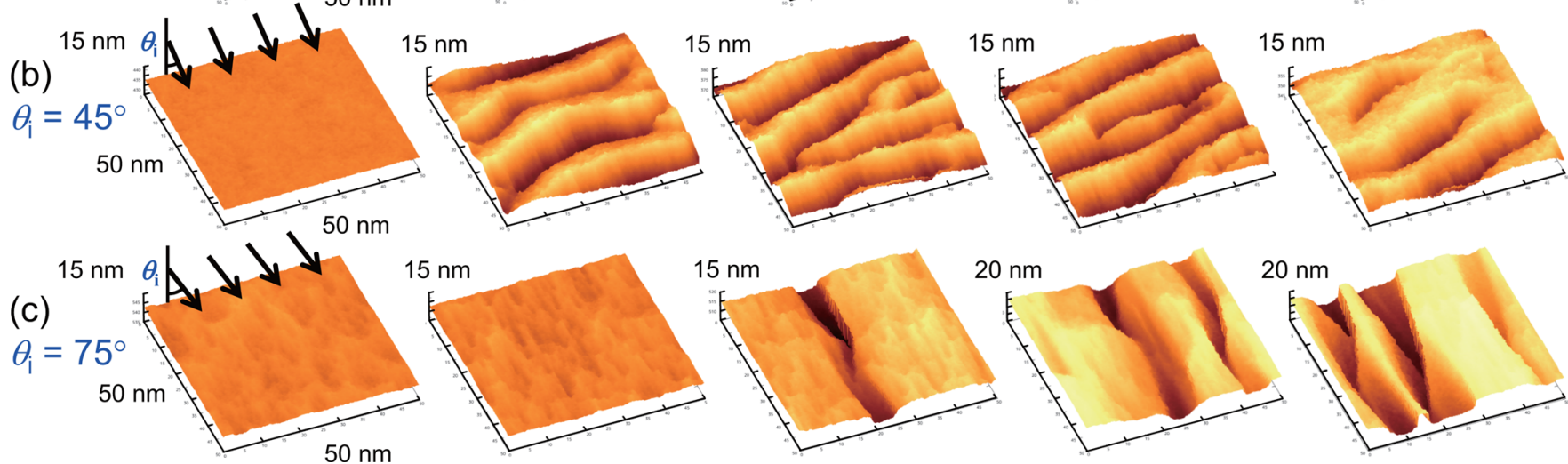

FIG. 7. (Color online) Surface features of $\mathrm{Si}$ at $t=60 \mathrm{~s}$ after the start of etching in $\mathrm{Cl}_{2}$ plasma, simulated for different neutral-to-ion flux ratios in the range $\Gamma_{n}^{0} / \Gamma_{i}^{0}=10-500$ with ion incidence angles of $\theta_{i}=$ (a) $0^{\circ}$, (b) $45^{\circ}$, and (c) $75^{\circ}$. The other calculation conditions in the ASCeM-3D are the same as those of Fig. 3 $\left(E_{i}=100 \mathrm{eV}, \Gamma_{o}{ }^{0}=\Gamma_{p}{ }^{0}=0\right)$. Note that the surface features for $\Gamma_{n}{ }^{0} / \Gamma_{i}^{0}=100$ of interest here are the same as those shown at $t=60 \mathrm{~s}$ in Figs. 3(a), 3(d), and 3(f).

model, or the known $\Gamma_{n}^{0} / \Gamma_{i}^{0}$ and angle dependences of the etch yield for ion-enhanced reactions. ${ }^{50,52,54}$

The rms roughness at $\theta_{i}=0^{\circ}$ peaks at $\Gamma_{n}^{0} / \Gamma_{i}^{0} \approx 50$ and then decreases with increasing $\Gamma_{n}^{0} / \Gamma_{i}^{0}$. It is noted here that at $\theta_{i}=0^{\circ}$, the decrease in roughness with $\Gamma_{n}^{0} / \Gamma_{i}^{0}$ is consistent with the results of some plasma etching experiments of blank $\mathrm{Si}$ substrates in $\mathrm{SF}_{6}$ previously reported, ${ }^{9}$ while is inconsistent with those of some other plasma experiments of Si etching in $\mathrm{SF}_{6}$ (Ref. 26) and $\mathrm{Cl}_{2}$ (Ref. 25); in effect, the experiments showed a decrease ${ }^{9}$ and an increase ${ }^{25,26}$ in roughness with increasing feed gas pressure. The decrease in roughness with increasing $\Gamma_{n}^{0} / \Gamma_{i}^{0}$ at $\theta_{i}=0^{\circ}$ is considered to be due to increased chemical effects (chemical and ion-enhanced etching reactions) as compared to those of sputtering. The rms roughness at $\theta_{i}=45^{\circ}$ increases with increasing $\Gamma_{n}^{0} / \Gamma_{i}^{0}$ and then tends to remain almost constant (a)

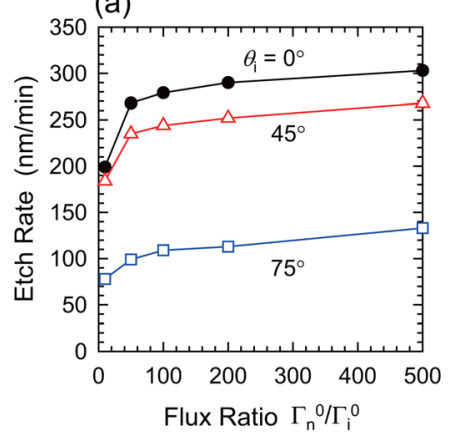

(b)

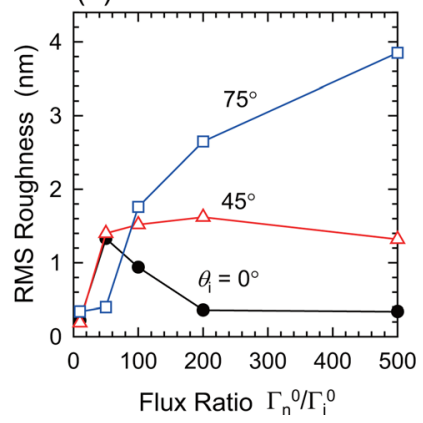

FIG. 8. (Color online) (a) Etch rate and (b) rms surface roughness at $t=60 \mathrm{~s}$ as a function of neutral-to-ion flux ratio in the range $\Gamma_{n}^{0} / \Gamma_{i}^{0}=10-500$ with ion incidence angles of $\theta_{i}=0^{\circ}, 45^{\circ}$, and $75^{\circ}$ derived from the simulations of Fig. 7. for further increased $\Gamma_{n}^{0} / \Gamma_{i}^{0}>50$. In Fig. $7(\mathrm{~b})$, the ripple structure at $\theta_{i}=45^{\circ}$ is seen to be significant in the range $\Gamma_{n}^{0} / \Gamma_{i}^{0} \approx 50-200$, while it tends to fade away for further increased $\Gamma_{n}^{0} / \Gamma_{i}^{0}$; in addition, the ripple wavelength at $\theta_{i}=45^{\circ}$ is the shortest for $\Gamma_{n}^{0} / \Gamma_{i}^{0} \approx 100$, being about $13 \mathrm{~nm}$. On the other hand, the rms roughness at $\theta_{i}=75^{\circ}$ increases gradually and then significantly with increasing $\Gamma_{n}{ }^{0} / \Gamma_{i}^{0}$; correspondingly, in Fig. 7(c), the ripple at $\theta_{i}=75^{\circ}$ is seen to enlarge significantly for increased $\Gamma_{n}^{0} / \Gamma_{i}^{0}>100$, resulting in a few wide, deep grooves, similarly to the roughened surface features at $\theta_{i}=75^{\circ}$ for $E_{i}>100 \mathrm{eV}$ in Fig. 5(c).

\section{Effects of oxygen flux $\left(\Gamma_{o}^{0} \Gamma_{i}^{0}\right)$}

Figure 9 shows the surface features of $\mathrm{Si}$ at $t=60 \mathrm{~s}$ after the start of etching in $\mathrm{Cl}_{2} / \mathrm{O}_{2}$ plasma, simulated in the presence of incoming oxygen fluxes from the plasma $\left(\Gamma_{o}^{0} \neq 0\right)$ with ion incidence angles of $\theta_{i}=0^{\circ}, 45^{\circ}$, and $75^{\circ}$. The oxygen-to-ion flux ratio concerned is in the range $\Gamma_{o}^{0} / \Gamma_{i}^{0}$ $=0-0.5$, and the other calculation conditions in the ASCeM-3D are the same as those of Fig. $3\left(E_{i}=100 \mathrm{eV}\right.$, $\left.\Gamma_{n}{ }^{0} / \Gamma_{i}^{0}=100, \Gamma_{p}{ }^{0}=0\right)$. Note that the surface features for $\Gamma_{o}^{0} / \Gamma_{i}^{0}=0$ of interest here are the same as those shown at $t=60 \mathrm{~s}$ in Figs. 3(a), 3(d), and 3(f), and the corresponding etch rate and rms surface roughness are shown in Fig. 10 as a function of $\Gamma_{o}^{0} / \Gamma_{i}^{0}$. The etch rate decreases substantially with adding oxygen fluxes $\Gamma_{o}^{0} / \Gamma_{i}^{0}$ at any incidence angle $\theta_{i}$ owing to surface oxidation; moreover, for a given $\Gamma_{o}^{0} / \Gamma_{i}^{0}>0$, the etch rate is smaller at $\theta_{i}=0^{\circ}$ and $45^{\circ}$ than at $\theta_{i}=75^{\circ}$. These reflect the etching or sputtering characteristics of oxidized Si taken in the model, or the known $\Gamma_{o}^{0} / \Gamma_{i}^{0}$ and angle dependences of the etch or sputter yield for energetic ions on oxidized surfaces. ${ }^{51,56}$ In effect, the etch yield 


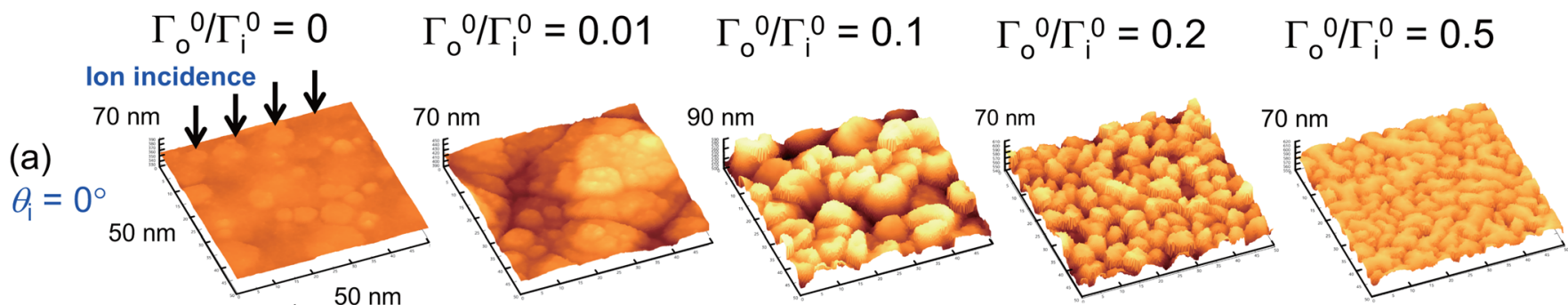

(b)
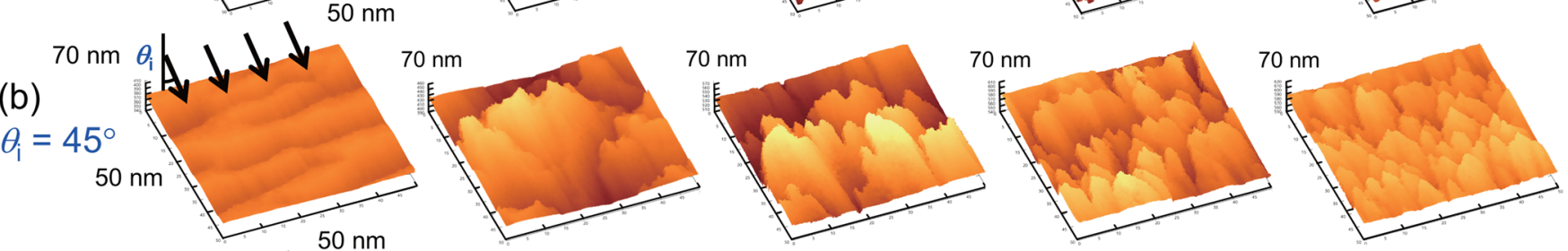

(c)
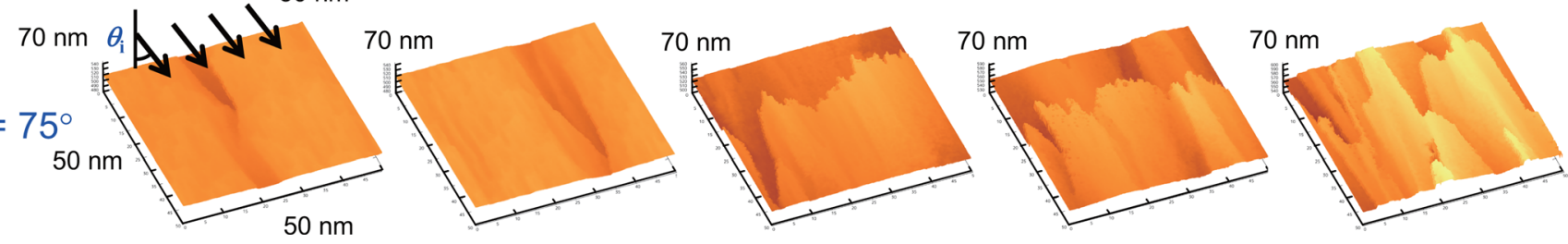

FIG. 9. (Color online) Surface features of $\mathrm{Si}$ at $t=60 \mathrm{~s}$ after the start of etching in $\mathrm{Cl}_{2} / \mathrm{O}_{2}$ plasma, simulated in the presence of incoming oxygen fluxes from the plasma $\left(\Gamma_{o}{ }^{0} \neq 0\right.$ ) with ion incidence angles of $\theta_{i}=$ (a) $0^{\circ}$, (b) $45^{\circ}$, and (c) $75^{\circ}$. The oxygen-to-ion flux ratio concerned is in the range $\Gamma_{o}^{0} / \Gamma_{i}^{0}=0-0.5$, and the other calculation conditions in the ASCeM-3D are the same as those of Fig. $3\left(E_{i}=100 \mathrm{eV}, \Gamma_{n}^{0} / \Gamma_{i}^{0}=100, \Gamma_{p}{ }^{0}=0\right)$. Note that the surface features for $\Gamma_{o}^{0} / \Gamma_{i}^{0}=0$ of interest here are the same as those shown at $t=60 \mathrm{~s}$ in Figs. 3(a), 3(d), and 3(f).

for ion-enhanced reactions is known to be maximum at $\theta_{i}=0^{\circ}$, decreasing with increasing $\theta_{i},{ }^{50,52,54}$ while the sputter yield peaks at $\theta_{i} \approx 60^{\circ}-70^{\circ} ;{ }^{51,56}$ in addition, at $\theta_{i}=0^{\circ}$ for $\mathrm{Cl}^{+}$ions of $E_{i}=100 \mathrm{eV}$, the ion-enhanced etch yield on $\mathrm{Si}$ surfaces chlorine saturated is known to be about eight times larger than the sputter yield on $\mathrm{SiO}_{2}{ }^{57}$

The rms roughness at $\theta_{i}=0^{\circ}$ and $45^{\circ}$ increases with increasing $\Gamma_{o}^{0} / \Gamma_{i}^{0}$, peaks at $\Gamma_{o}^{0} / \Gamma_{i}^{0} \approx 0.1$, and then decreases. It is noted here that at $\theta_{i}=0^{\circ}$, the increase in roughness with $\Gamma_{o}^{0} / \Gamma_{i}^{0}$ is consistent with the results of plasma etching experiments of blank Si substrates in $\mathrm{Cl}_{2} / \mathrm{O}_{2}$ previously reported, ${ }^{84}$ which showed an increase in roughness with increasing $\mathrm{O}_{2}$ flux added; this is attributed to local surface oxidation or the local formation of oxide micromasks during etching. Moreover, in Fig. 9(a), the roughened surface (a)

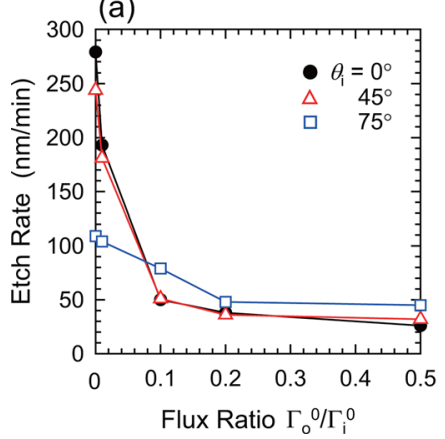

(b)

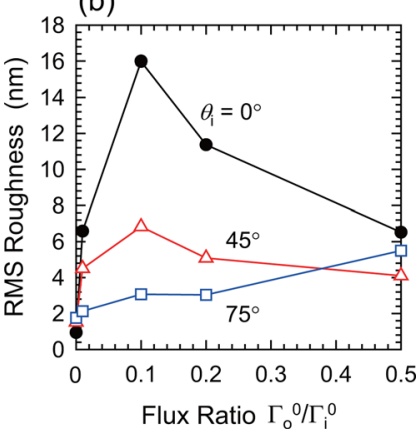

FIG. 10. (Color online) (a) Etch rate and (b) rms surface roughness at $t=60 \mathrm{~s}$ as a function of oxygen-to-ion flux ratio in the range $\Gamma_{o}^{0} / \Gamma_{i}^{0}=0-0.5$ with ion incidence angles of $\theta_{i}=0^{\circ}, 45^{\circ}$, and $75^{\circ}$ derived from the simulations of Fig. 9. features at $\theta_{i}=0^{\circ}$ exhibit nanoscale columnar structures like pillars for increased $\Gamma_{o}^{0} / \Gamma_{i}^{0}>0.1$, as has been observed in some $\mathrm{O}_{2}$-containing plasma etching experiments of $\mathrm{Si}$ in $\mathrm{HCl} / \mathrm{O}_{2} / \mathrm{BCl}_{3},{ }^{85} \mathrm{HBr} / \mathrm{NF}_{3} / \mathrm{O}_{2},{ }^{86,87}$ and $\mathrm{SF}_{6} / \mathrm{O}_{2}{ }^{88}$ In Fig. $9(\mathrm{~b})$, the ripple structure at $\theta_{i}=45^{\circ}$ is seen to be the most significant for $\Gamma_{o}^{0} / \Gamma_{i}^{0}=0$ or in the absence of oxygen fluxes, and it fades away for increased $\Gamma_{o}^{0} / \Gamma_{i}^{0}>0$ with the appearance of squamous features that are analogous to those on roughened surfaces at $\theta_{i}=45^{\circ}$ for $E_{i}=500 \mathrm{eV}$ in Fig. 5(b). On the other hand, the rms roughness at $\theta_{i}=75^{\circ}$ continues to increase gradually with increasing $\Gamma_{o}^{0} / \Gamma_{i}^{0}$; correspondingly, in Fig. 9(c), the ripple at $\theta_{i}=75^{\circ}$ is seen to be the most significant for $\Gamma_{o}^{0} / \Gamma_{i}^{0} \approx 0-0.01$ and tends to fade away for further increased $\Gamma_{o}^{0} / \Gamma_{i}^{0}$ with the appearance of squamous features. However, it should be noted that the roughened surface features at $\theta_{i}=75^{\circ}$ for $\Gamma_{o}^{0} / \Gamma_{i}^{0}>0$ or in the presence of oxygen fluxes do not exhibit wide, deep grooves that occur at $\theta_{i}=75^{\circ}$ for $E_{i}>100 \mathrm{eV}$ in Fig. 5(c) and for $\Gamma_{n}{ }^{0} / \Gamma_{i}{ }^{0}>100$ in Fig. 7(c).

\section{Effects of etch byproduct flux $\left(\Gamma_{p}{ }^{0} \Lambda_{i}^{0}\right)$}

Figure 11 shows the surface features of $\mathrm{Si}$ at $t=60 \mathrm{~s}$ after the start of etching in $\mathrm{Cl}_{2}$ plasma, simulated in the presence of incoming etch/sputter byproduct fluxes from the plasma $\left(\Gamma_{p}^{0} \neq 0\right)$ with ion incidence angles of $\theta_{i}=0^{\circ}, 45^{\circ}$, and $75^{\circ}$. The returning probability concerned is in the range $P_{r}=0-0.5$, and the other calculation conditions in the ASCeM-3D are the same as those of Fig. $3\left(E_{i}=100 \mathrm{eV}\right.$, $\left.\Gamma_{n}^{0} / \Gamma_{i}^{0}=100, \Gamma_{o}^{0}=0\right)$. Note that the surface features for $P_{r}=0$ or $\Gamma_{p}^{0}=0$ of interest here are the same as those shown at $t=60 \mathrm{~s}$ in Figs. 3(a), 3(d), and 3(f), and the 


$$
\begin{array}{lllll}
P_{\mathrm{r}}=0 & P_{\mathrm{r}}=0.05 & P_{\mathrm{r}}=0.1 & P_{\mathrm{r}}=0.2 & P_{\mathrm{r}}=0.5
\end{array}
$$
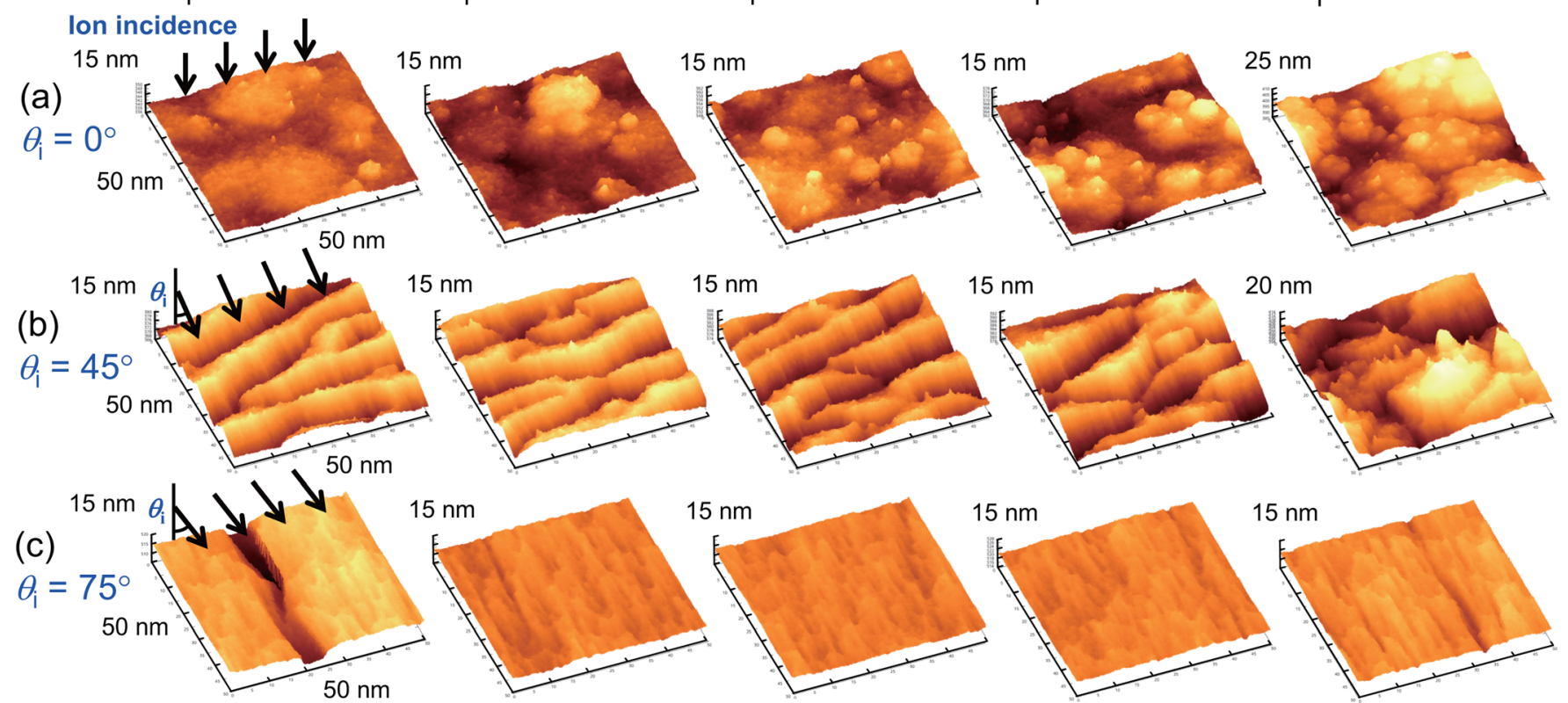

Fig. 11. (Color online) Surface features of $\mathrm{Si}$ at $t=60 \mathrm{~s}$ after the start of etching in $\mathrm{Cl}_{2}$ plasma, simulated in the presence of incoming etch/sputter byproduct fluxes from the plasma $\left(\Gamma_{p}^{0} \neq 0\right.$ ) with ion incidence angles of $\theta_{i}=$ (a) $0^{\circ}$, (b) $45^{\circ}$, and (c) $75^{\circ}$. The returning probability concerned is in the range $P_{r}=0-0.5$, and the other calculation conditions in the ASCeM-3D are the same as those of Fig. $3\left(E_{i}=100 \mathrm{eV}, \Gamma_{n}^{0} / \Gamma_{i}^{0}=100, \Gamma_{o}{ }^{0}=0\right)$. Note that the surface features for $P_{r}=0$ of interest here are the same as those shown at $t=60 \mathrm{~s}$ in Figs. 3(a), 3(d), and 3(f). It is further noted that $P_{r}$ is the probability assumed for the return of the etch/ sputter products that have been desorbed from feature surfaces into vacuum and then gone out of the simulation domain (or gone out into the plasma) during etching; e.g., in the case of $\theta_{i}=0^{\circ}$ under the present calculation conditions, the probabilities in the range $P_{r}=0-0.5$ correspond to the ratios $\Gamma_{p}^{0} / \Gamma_{i}^{0} \approx 0-1.0$ of the byproduct to ion fluxes coming from the plasma onto substrate surfaces during etching.

corresponding etch rate and rms surface roughness are shown in Fig. 12 as a function of $P_{r}$. It is further noted that in the case of $\theta_{i}=0^{\circ}$ under these conditions, e.g., the respective probabilities $P_{r}=0.05,0.1,0.2$, and 0.5 correspond to the ratios $\Gamma_{p}{ }^{0} / \Gamma_{i}^{0} \approx 0.10,0.21,0.41$, and 1.0 of the byproduct to ion fluxes coming from the plasma onto substrate surfaces during etching. ${ }^{42}$ The etch rate decreases slightly with increasing returning probability $P_{r}$ or byproduct flux $\Gamma_{p}^{0} / \Gamma_{i}^{0}$ at any incidence angle $\theta_{i}$; moreover, for a given $P_{r}$ or $\Gamma_{p}^{0} / \Gamma_{i}^{0}$, the etch rate is larger at $\theta_{i}=0^{\circ}$ and $45^{\circ}$ than at $\theta_{i}=75^{\circ}$. These reflect the ion-enhanced etching characteristics of Si taken in the model with light deposition of etch/sputter byproducts on surfaces being etched, ${ }^{42}$ where the (a)

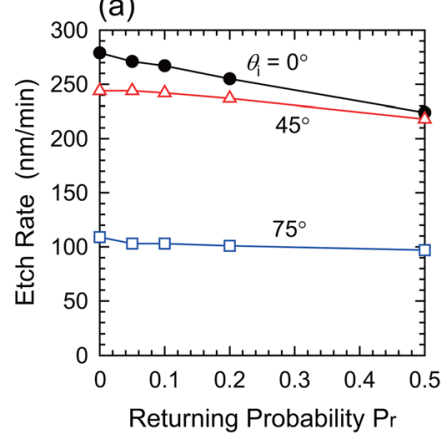

(b)

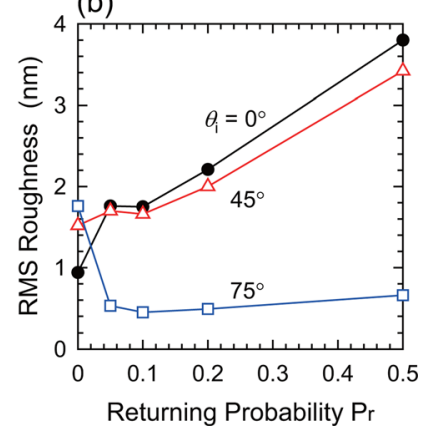

FIG. 12. (Color online) (a) Etch rate and (b) rms surface roughness at $t=60 \mathrm{~s}$ as a function of returning probability in the range $P_{r}=0-0.5$ with ion incidence angles of $\theta_{i}=0^{\circ}, 45^{\circ}$, and $75^{\circ}$ derived from the simulations of Fig. 11. etch yield has energy and angle dependences similar to those for ion-enhanced reactions without deposition.

The rms roughness at $\theta_{i}=0^{\circ}$ and $45^{\circ}$ increases with increasing $P_{r}$ or $\Gamma_{p}^{0} / \Gamma_{i}^{0}$. It is noted here that at $\theta_{i}=0^{\circ}$, the increase in roughness with $\Gamma_{p}^{0} / \Gamma_{i}^{0}$ is consistent with the results of 2D (Ref. 23) and 3D (Ref. 31) cellular Monte Carlo simulations of $\mathrm{SF}_{6}$ plasma etching of $\mathrm{Si}$, which showed an increase in roughness in the presence of incoming fluxes of byproducts or etch inhibitors; this is attributed to local byproduct deposition or the local formation of byproduct micromasks on surfaces during etching. In Fig. 11(b), the ripple structure at $\theta_{i}=45^{\circ}$ is seen to be the most significant for $\Gamma_{p}{ }^{0} / \Gamma_{i}^{0}=0$ or in the absence of byproduct fluxes, and it tends to fade away for increased $\Gamma_{p}^{0} / \Gamma_{i}^{0}>0$ with the ripple wavelength of about $13 \mathrm{~nm}$ remaining almost unchanged, although the rms roughness increases with increasing $\Gamma_{p}^{0} / \Gamma_{i}^{0}$. On the other hand, the rms roughness at $\theta_{i}=75^{\circ}$ decreases substantially with adding byproduct fluxes $\Gamma_{p}^{0} / \Gamma_{i}^{0}$; correspondingly, in Fig. 11(c), the ripple at $\theta_{i}=75^{\circ}$ is seen to be the most significant for $\Gamma_{p}^{0} / \Gamma_{i}^{0}=0$ and tends to be less significant for increased $\Gamma_{p}^{0} / \Gamma_{i}^{0}>0$. It should be noted that the roughened surface features at $\theta_{i}=75^{\circ}$ for $\Gamma_{p}^{0} / \Gamma_{i}^{0}>0$ or in the presence of byproduct fluxes do not exhibit wide, deep grooves, similarly to the surface features at $\theta_{i}=75^{\circ}$ for $\Gamma_{o}^{0} / \Gamma_{i}^{0}>0$ or with oxygen fluxes in Fig. $9(\mathrm{c})$. The decrease in roughness and ripple with increasing $P_{r}$ or $\Gamma_{p}^{0} / \Gamma_{i}^{0}$ at $\theta_{i}=75^{\circ}$ would be consistent with plasma etching processes to suppress the LER and LWR on feature sidewalls for pattern definition through etch inhibitor deposition or passivation layer formation thereon. ${ }^{32,36}$ 


\section{Power spectral density analysis}

To further characterize the roughened surface features, the power spectral density (PSD) distribution as well as rms roughness of feature surfaces is important, ${ }^{9,25,26}$ because the rms roughness reflects only the vertical extent of the feature, while the PSD gives the information on both its vertical and lateral (or spatial) extent. In the PSD analysis, the 2D PSD (2D-PSD) function $P\left(k_{x}, k_{y}\right)$ at time $t$ is calculated from the 2D discrete Fourier transform (2D-DFT) of the 2D surface height or depth distribution $z(m, n)$ in the $(x, y)$ coordinates $^{89-92}$

$$
\begin{aligned}
P\left(k_{x}, k_{y}\right)= & \frac{1}{W^{2}} \mid \sum_{m=1}^{N} \sum_{n=1}^{N} z(m, n) \\
& \times\left.\exp \left[-2 \pi j \Delta W\left(k_{x} m+k_{y} n\right)\right](\Delta W)^{2}\right|^{2},
\end{aligned}
$$

where the symbols $W=50 \mathrm{~nm}, N=185, m, n$, and $z(m, n)$ are the same as those in Eq. (1), $j$ is the imaginary unit, $k_{x}$ and $k_{y}$ are the spatial frequency in the $x$ - and $y$-directions, and $\Delta W=W / N=L=2.7 \AA$ is the ASCeM cell size or the spatial dimension for analysis. The calculation is further followed by the integration or discrete sum over one of the two variables in the 2D-PSD to give the one-dimensional PSD (1D-PSD) function at time $t^{89,91}$

$$
P_{x}\left(k_{x}\right)=\sum_{k_{y}} P\left(k_{x}, k_{y}\right) \Delta k_{y},
$$

where $P_{x}\left(k_{x}\right)$ is the $1 \mathrm{D}$-PSD along the $x$ axis, $\Delta k_{y}=1 / W$ is the spatial frequency dimension for analysis, and $P_{y}\left(k_{y}\right)$ along the $y$ axis is similarly given with $\Delta k_{x}=1 / W$. Note that the 1D-PSD function has a unit of third power to the length, while the 2D-PSD has a unit of fourth power to the length.
Figure 13 shows the time evolution of the 1D-PSD distributions $P_{x}\left(k_{x}\right)$ and $P_{y}\left(k_{y}\right)$ along the $x$ - and $y$-axes for an ion incidence angle $\theta_{i}=0^{\circ}$, extracted from that of the 2D-PSD of the surface features of $\mathrm{Si}$ etched in $\mathrm{Cl}_{2}$ plasma shown in the ASCeM-3D simulations of Fig. 3(a) $\left(E_{i}=100 \mathrm{eV}\right.$, $\left.\Gamma_{n}^{0} / \Gamma_{i}^{0}=100, \Gamma_{o}{ }^{0}=\Gamma_{p}{ }^{0}=0\right)$. Also shown in the figure are the corresponding $2 \mathrm{D}$ images of the surface features simulated, where the ion incidence is in the negative $z$ direction. The length scale concerned is the side length $W=50 \mathrm{~nm}$ of the $2 \mathrm{D}$ square cross section of the ASCeM-3D simulation domain with the cell size $L=2.7 \AA(=0.27 \mathrm{~nm})$, which corresponds to the spatial frequency ranging from $k_{x}, k_{y}=0.02$ to $3.7 \mathrm{~nm}^{-1}$ in the reciprocal space. For the 1D-PSD curve, the parameters of interest are $9,25,26$ the plateau height value $w_{\alpha 0}$ at smaller $k_{\alpha}$ related to the vertical height or depth of roughened surface features $\left[P_{\alpha}\left(k_{\alpha}\right) \approx w_{\alpha 0}\right.$ at $\left.k_{\alpha}<k_{\alpha 0}\right]$, and the correlation length $\xi_{\alpha 0}\left(=1 / k_{\alpha 0}\right)$ that defines the lateral size of the roughness; moreover, the behavior at large $k_{\alpha}$ gives the fractal or self-affine nature of the roughness $\left[P_{\alpha}\left(k_{\alpha}\right)\right.$ $\propto 1 / k_{\alpha}{ }^{\nu_{\alpha}}$ at $\left.k_{\alpha}>k_{\alpha 0}\right],{ }^{92}$ where the exponent $\nu_{\alpha}$ is a constant linked to the fractal dimension of the surface, and $\alpha=x$ and $y$ are for $P_{x}\left(k_{x}\right)$ and $P_{y}\left(k_{y}\right)$, respectively.

The analysis indicates that at $\theta_{i}=0^{\circ}$ or normal incidence, the formation and evolution of the surface roughness is random or isotropic, and correspondingly, the $P_{x}\left(k_{x}\right)$ and $P_{y}\left(k_{y}\right)$ curves are similar at any time: the plateau value $w_{\alpha 0}$ and correlation length $\xi_{\alpha 0}$ increase with time, corresponding to the increase in both vertical and lateral roughness, which is consistent with the results of plasma etching experiments of blank $\mathrm{Si}$ substrates in $\mathrm{SF}_{6}$ previously reported. ${ }^{9}$ Note that the values of $w_{\alpha 0}$ and $\xi_{\alpha 0}$ tend to become unclear with time, which is ascribed to the limit length scale $W=50 \mathrm{~nm}$ or its reciprocal $k_{\alpha}=0.02 \mathrm{~nm}^{-1}$ in the present analysis. In addition, the $P_{x}\left(k_{x}\right)$ and $P_{y}\left(k_{y}\right)$ tails at high spatial frequencies remain almost unchanged with time, exhibiting a fractal-linked constant $\nu_{\alpha} \approx 1$ (extreme fractal) in the range $0.2<k_{\alpha}<2 \mathrm{~nm}^{-1}$.

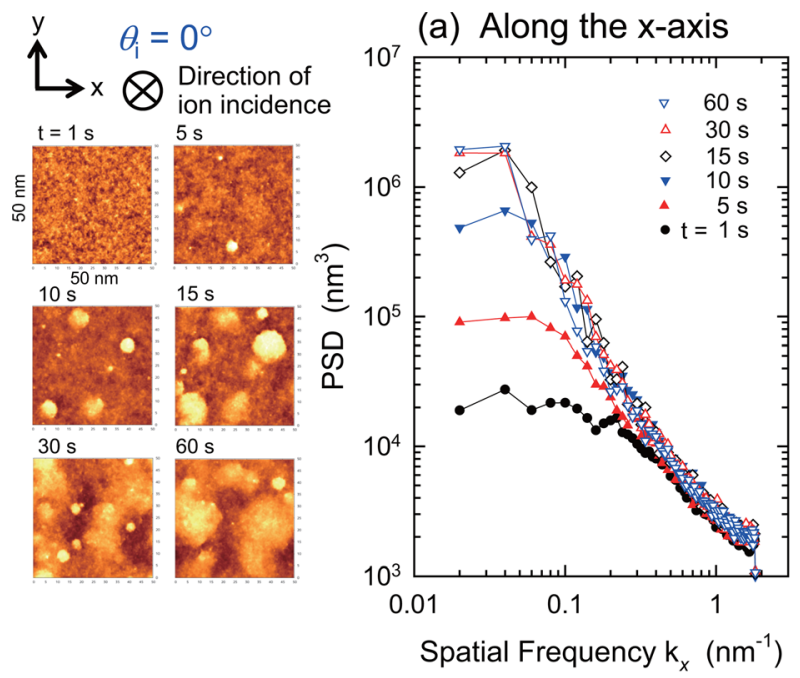

(b) Along the y-axis

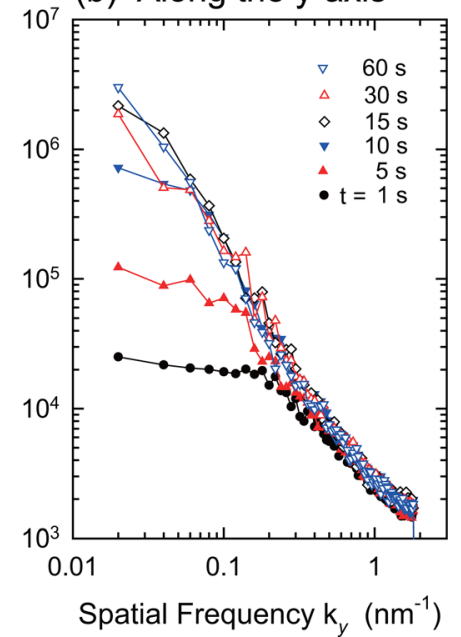

FIG. 13. (Color online) Time evolution of the 1D-PSD distributions of (a) $P_{x}\left(k_{x}\right)$ along the $x$-axis and (b) $P_{y}\left(k_{y}\right)$ along the $y$-axis for an ion incidence angle $\theta_{i}=0^{\circ}$ (normal incidence), extracted from that of the 2D-PSD of the surface features of Si etched in $\mathrm{Cl}_{2}$ plasma shown in the ASCeM-3D simulations of Fig. 3(a) $\left(E_{i}=100 \mathrm{eV}, \Gamma_{n}{ }^{0} / \Gamma_{i}{ }^{0}=100, \Gamma_{o}{ }^{0}=\Gamma_{p}{ }^{0}=0\right)$. Also shown are the corresponding 2D images of the surface features simulated, where the ion incidence is in the negative $z$ direction. The length scale concerned is the side length $W=50 \mathrm{~nm}$ of the $2 \mathrm{D}$ square cross section of the ASCeM-3D simulation domain with the cell size $L=2.7 \AA$ ( $=0.27 \mathrm{~nm})$, which corresponds to the spatial frequency ranging from $k_{x}, k_{y}=0.02$ to $3.7 \mathrm{~nm}^{-1}$ in the reciprocal space. 


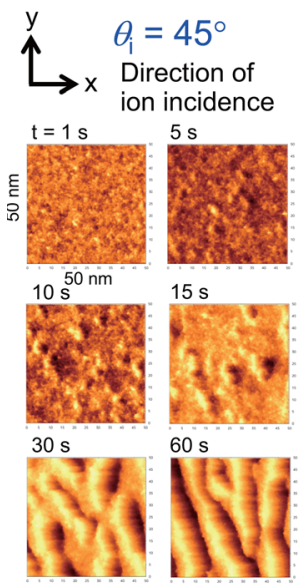

(a) Along the x-axis

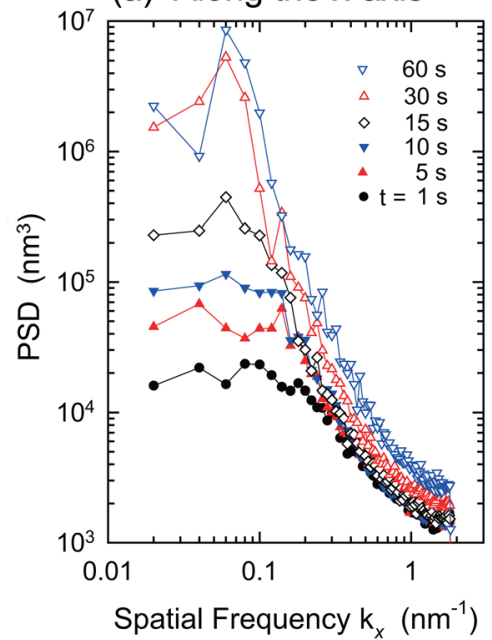

(b) Along the y-axis

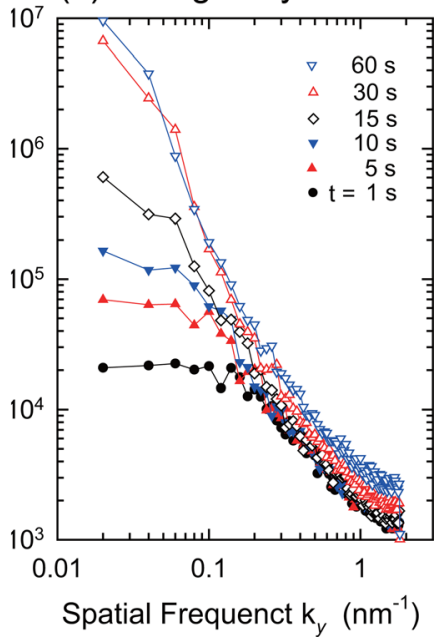

FIG. 14. (Color online) Time evolution of the 1D-PSD distributions of (a) $P_{x}\left(k_{x}\right)$ along the $x$-axis and (b) $P_{y}\left(k_{y}\right)$ along the $y$-axis for an ion incidence angle $\theta_{i}=45^{\circ}$ (oblique incidence), extracted from that of the 2D-PSD of the surface features of Si etched in $\mathrm{Cl}_{2}$ plasma shown in the ASCeM-3D simulations of Fig. 3(d). Also shown are the corresponding 2D images of the surface features simulated, where the ion incidence is in the $x$ direction, and the other figure properties are the same as those of Fig. 13.

This implies that the increase in roughness with time arises primarily from an increase in height/depth of lowerfrequency (or longer-wavelength) components, without a significant change in height/depth of high-frequency (or short-wavelength) components.

Figures 14 and 15 show the time evolution of the 1D-PSD distributions $P_{x}\left(k_{x}\right)$ and $P_{y}\left(k_{y}\right)$ along the $x$ - and $y$-axes for ion incidence angles $\theta_{i}=45^{\circ}$ and $75^{\circ}$, respectively, also extracted from that of the 2D-PSD of the surface features of $\mathrm{Si}$ etched in $\mathrm{Cl}_{2}$ plasma shown in the ASCeM-3D simulations of Figs. 3(d) and 3(f). The $x$ - and $y$-directions shown correspond to that parallel and perpendicular to the direction of ion incidence, respectively, and the other figure properties are the same as those of Fig. 13 for $\theta_{i}=0^{\circ}$. The analysis indicates that at increased $\theta_{i}=45^{\circ}$ or oblique incidence, the
$P_{x}\left(k_{x}\right)$ and $P_{y}\left(k_{y}\right)$ curves are similar at early times $(t<5 \mathrm{~s})$, implying that the formation and evolution of the roughness is then random or isotropic; in particular, the $P_{x}\left(k_{x}\right)$ and $P_{y}\left(k_{y}\right)$ curves soon after the start of etching $(t=1 \mathrm{~s})$ are almost the same as those at $\theta_{i}=0^{\circ}$. On the other hand, the evolutions of $P_{x}\left(k_{x}\right)$ and $P_{y}\left(k_{y}\right)$ are not similar thereafter $(t>10 \mathrm{~s})$, when $P_{x}\left(k_{x}\right)$ exhibits a local apparent maximum at the spatial frequency $k_{x} \approx 0.06 \mathrm{~nm}^{-1}$, corresponding to the periodic or ripple structures of wavelength $\lambda_{x}=1 / k_{x} \approx$ $16 \mathrm{~nm}$ that evolve perpendicularly to the direction of ion incidence.

The analysis further indicates that at further increased $\theta_{i}=75^{\circ}$ or grazing incidence, the evolutions of $P_{x}\left(k_{x}\right)$ and $P_{y}\left(k_{y}\right)$ are not similar at early as well as later times; in effect, soon after the start of etching $(t=1 \mathrm{~s})$, the plateau value is
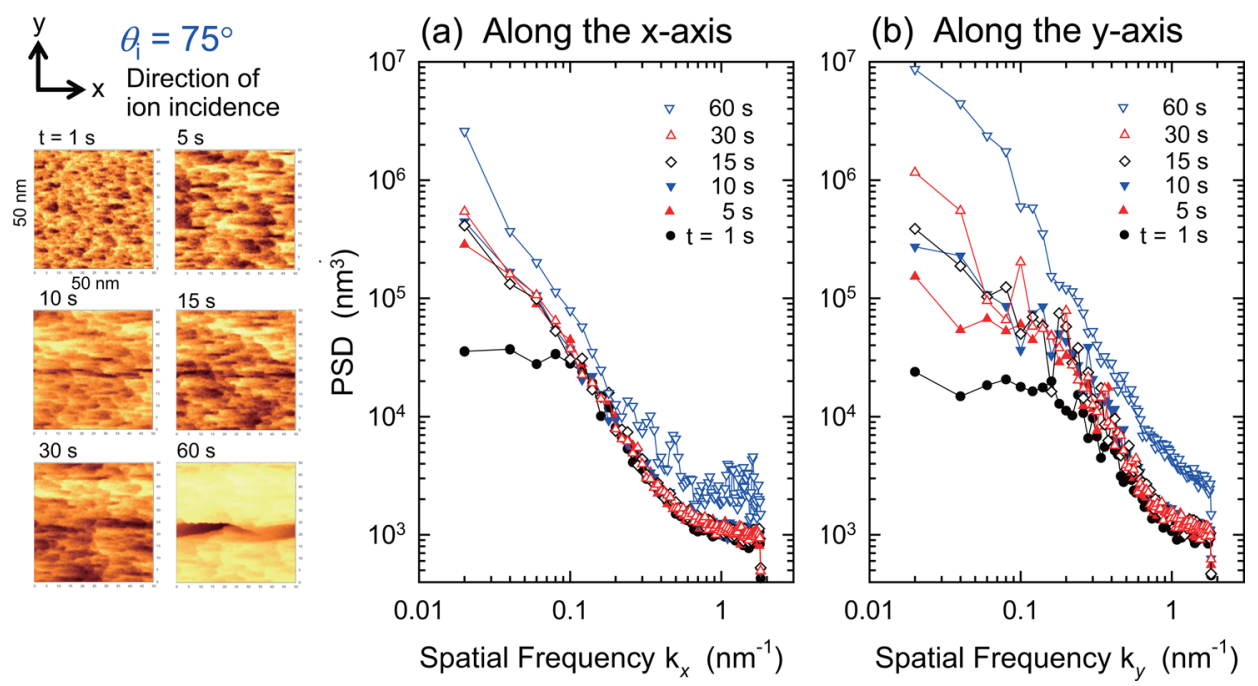

FIg. 15. (Color online) Time evolution of the 1D-PSD distributions of (a) $P_{x}\left(k_{x}\right)$ along the $x$-axis and (b) $P_{y}\left(k_{y}\right)$ along the $y$-axis for an ion incidence angle $\theta_{i}=75^{\circ}$ (grazing incidence), extracted from that of the 2D-PSD of the surface features of Si etched in $\mathrm{Cl}_{2}$ plasma shown in the ASCeM-3D simulations of Fig. 3(f). Also shown are the corresponding 2D images of the surface features simulated, where the ion incidence is in the $x$ direction, and the other figure properties are the same as those of Fig. 13. 
larger and the correlation length is smaller along the $x$-axis than along the $y$-axis $\left(w_{x 0}>w_{y 0}, \xi_{x 0}<\xi_{y 0}\right)$, and $P_{y}\left(k_{y}\right)$ exhibits small maxima at spatial frequencies in the range $0.2<k_{y}<0.5 \mathrm{~nm}^{-1}$, implying that small grooves elongated parallel to the direction of ion incidence tend to occur at relatively early times. Moreover, at later times $(t>5 \mathrm{~s}), P_{x}\left(k_{x}\right)$ remains almost unchanged with time, while $P_{y}\left(k_{y}\right)$ exhibits several maxima at frequencies in the range not only $0.2<k_{y}<0.5 \mathrm{~nm}^{-1}$ but also $0.1<k_{y}<0.2 \mathrm{~nm}^{-1}$; this corresponds to small ripples or slitlike grooves of wavelengths in the range $2<\lambda_{y}=1 / k_{y}<10 \mathrm{~nm}$, which evolve parallel to the direction of ion incidence without a significant change in roughness along the direction of ion incidence.

\section{Mechanisms for surface roughening and rippling}

Surface roughening is generally assumed to be 3D and stochastic. $^{24,28}$ The ASCeM-3D is a 3D Monte Carlo-based simulation model for plasma-surface interactions and the feature profile evolution during plasma etching. Thus, in the ASCeM-3D, stochastic processes are intrinsically taken into account through using random numbers in the Monte Carlo calculation of the interaction of ions and neutrals with substrate surfaces (surface chemistry and kinetics); random numbers are also used in the determination of incoming parameters of ions and neutrals injected from the top of the simulation domain (particle injection position, time, energy, and angle). On nanometer scale, ions and neutrals incident on surfaces are few in number during etching; e.g., the number of incident ions is estimated to be $\Gamma_{i}^{0} A \Delta T=60$ during etching substrate surfaces of $A=1 \mathrm{~nm}^{2}$ in a depth $D=1 \mathrm{~nm}$ with an etch rate $\mathrm{ER}=100 \mathrm{~nm} / \mathrm{min}$, where $\Gamma_{i}^{0}=1.0$ $\times 10^{16} \mathrm{~cm}^{-2} \mathrm{~s}^{-1}$ is the ion flux concerned in this study, and $\Delta T=D / \mathrm{ER}=0.6 \mathrm{~s}$ is the etching time. Therefore, it is probable that the low-level surface roughening at not only the initial (or induction) but also the following stage of plasma etching is stochastic at any angle $\theta_{i}$ of ion incidence, as seen above in the PSD analysis of Figs. 13-15; in practice, the stochastic roughening continues to occur during etching at any $\theta_{i}$, originating from the temporal as well as spatial uniformity of the incident flux and angle of ions and/or neutral etchants on surfaces at microscale. However, the mechanisms for the evolution of surface roughness and ripples with time during etching are not yet fully understood.

A few mechanisms, such as geometrical shadowing, ${ }^{13}$ surface reemission of etchants, ${ }^{14-16,22}$ and effects of etch inhibitors, ${ }^{23,31}$ have so far been invoked to interpret the evolution of the surface roughness during plasma etching. The ASCeM-3D model takes into account all these surface chemistry and kinetics, as described in Sec. II A 3. Further investigations with the ASCeM-3D indicated that the surface reemission of etchants (depending on the sticking probability $S_{n}$ ) does not affect the evolution of roughness and ripples; on the other hand, the etch inhibitor deposition or passivation layer formation (depending on incoming fluxes $\Gamma_{o}^{0}$ and $\Gamma_{p}{ }^{0}$, and also on sticking probabilities $S_{o}$ and $S_{p}$ ) was found to affect significantly the evolution of roughness and ripples, as seen in Sec. II B 4 and Sec. II B 5. Note that the etch or surface inhibitors concerned in this study are oxygen and etch/sputter byproducts (hard and soft inhibitors, ${ }^{23,31}$ respectively) coming from the plasma, and it is probable that the local micromasking caused by local surface oxidation and byproduct deposition results in roughened surface features at microscale. In addition, the redeposition of etch/sputter products desorbed from feature surfaces being etched (depending on the sticking probability $S_{q}$ ) was also found to affect the evolution of roughness and ripples, which is ascribed probably to similar mechanisms.

The ASCeM-3D further indicated that the ion reflection or scattering from surfaces on incidence is crucial for surface roughening and rippling during plasma etching; in effect, it was found that in the absence of the effects of ion reflection (or assuming that all the ions incident on surfaces penetrate into substrates without reflection), the roughness is reduced substantially to a low level (RMS $\approx 0.2 \mathrm{~nm}$ ) and the ripple structures fade away, with the etch rate remaining almost unchanged. Moreover, the ASCeM-3D indicated that at increased surface temperatures $T_{s}$, the roughness is reduced and the ripples tend to disappear, with the etch rate being increased owing to effects of chemical etching reactions enhanced; in addition, it was found that widening the angular distribution of incoming ion fluxes (or decreasing the parameter $R=E_{i} / k T_{i}$ ) reduces the roughness and ripples without a significant change of the etch rate, while the energy distribution of ion fluxes (e.g., a bimodal ion energy distribution) does not affect the evolution of roughness and ripples. Therefore, it is also probable that the effects of the ion reflection or scattering from microscopically roughened feature surfaces on incidence is indispensable for the evolution of surface roughness and ripples during plasma etching, while the surface temperature (or chemical etching) and the ion angular distribution (or ion temperature) play a role in smoothing surfaces; in effect, the reflection of energetic ions from feature surfaces leads to the concentration of them onto the bottom of the feature, ${ }^{47}$ thus resulting in an increase in roughness to its vertical as well as lateral extent.

Mechanisms for surface rippling in response to ion incidence angle $\theta_{i}$ have so far been studied to interpret the experiments of the interaction of $\mathrm{keV}$ ion beams with surfaces, using a continuum model ${ }^{72,75,77,78,80-83}$ and Monte Carlo simulation. ${ }^{69,72,79}$ The ripple orientation and wavelength depending on $\theta_{i}$ were first predicted by a continuum model of Bradley and Harper $(\mathrm{B}-\mathrm{H}),{ }^{77}$ taking into account a competition between roughening caused by surface curvature-dependent sputtering and smoothing caused by surface diffusion of atoms. The $\mathrm{B}-\mathrm{H}$ model was then extended by adding a few mechanisms such as ion reflection or scattering, ${ }^{78}$ ion-induced surface diffusion, ${ }^{80}$ geometrical shadowing, ${ }^{81}$ and redeposition of sputtered atoms, ${ }^{83}$ to further interpret the ripple wavelength and amplitude depending on ion energy, ion flux, and surface temperature, along with the evolution of ion-sputtered surface features. The Monte Carlo simulation of beam-surface interactions was also utilized to interpret the experiments, including all the mechanisms taken in the B-H model. ${ }^{69,72,79}$ Similarly, the Monte Carlo-based ASCeM-3D model for plasma-surface 
interactions also takes into account most of the surface chemistry and kinetics that would occur, showing the formation and evolution of surface ripples during plasma etching in response to ion incidence angle $\theta_{i}$, as described above in this Sec. II B. The ASCeM-3D implied that the ripples perpendicular to the direction of ion incidence on surfaces being etched (typically at $\theta_{i}=45^{\circ}$ or oblique incidence) are caused by both effects of the ion reflection or scattering from feature surfaces on incidence and the locally different etch/sputter yields thereon; ${ }^{47}$ in practice, the local angle $\theta$ of ion incidence varies with the local position on microscopically roughened feature surfaces, thus giving locally different etch/sputter yields. The ripples parallel to the direction of ion incidence (typically at $\theta_{i} \geq 75^{\circ}$ or grazing incidence) are considered to be due to both effects of the ion reflection or scattering and the ion channeling on roughened feature surfaces, as previously suggested, ${ }^{24,28}$ where the reflection probability from surfaces on incidence increases with increasing incidence angle $\theta_{i} \cdot{ }^{41,47}$ However, a more relevant understanding of the mechanisms for surface rippling during plasma etching will require a further study including experimental demonstrations of it.

\section{EXPERIMENT}

\section{A. Experimental setup}

Figure 16 shows the experimental setup. The ICP plasma reactor consisted of a stainless-steel chamber $25 \mathrm{~cm}$ in diameter and $30 \mathrm{~cm}$ high, a three-turn planar coil on a dielectric window at the top, and a 4-in.-diameter electrode or wafer stage at the bottom. The discharge was established by $13.56-$ $\mathrm{MHz}$ rf powers of nominally $P_{\mathrm{ICP}}=450 \mathrm{~W}$. The wafer stage was capacitively coupled to a $13.56-\mathrm{MHz}$ rf power supply through an impedance matching network for additional $\mathrm{rf}$ biasing, where the rf bias power was varied in the range $P_{\text {rf }}=0-200 \mathrm{~W}$ to vary the ion incident energy $E_{i}$ onto substrate surfaces. The reactor chamber was evacuated to a base pressure $<10^{-6}$ Torr, and pure $\mathrm{Cl}_{2}$ gas was introduced therein at a pressure of $P_{0}=20$ mTorr with a mass flow rate of $F_{0}=20 \mathrm{sccm}$.

Samples for etching were 4-in.-diameter blank $\operatorname{Si}(100)$ wafers of $n$-type with a resistivity of $\rho \approx 10$ and $0.02 \Omega \cdot \mathrm{cm}$,

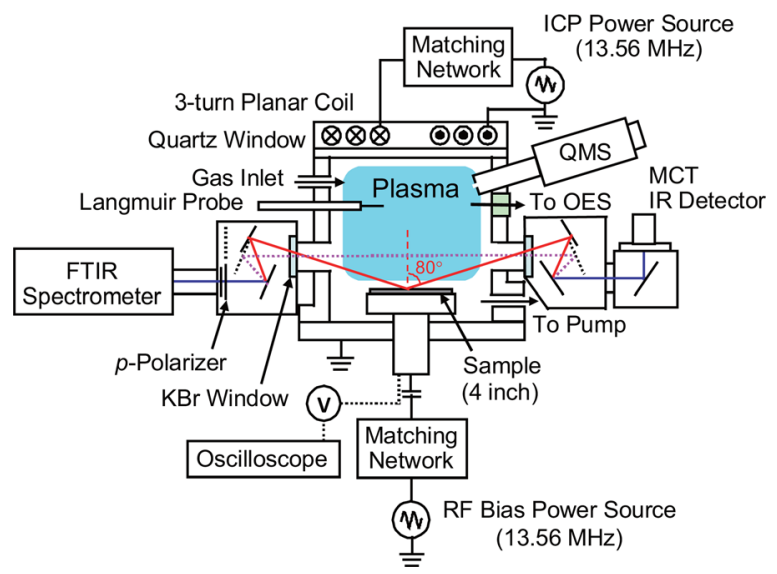

FIG. 16. (Color online) Experimental setup for plasma etching using an ICP reactor, including several plasma and surface diagnostics presently employed. which were pasted with silicone high vacuum grease into place on the wafer stage that was water cooled. The samples were cleaned through $\mathrm{HF}$ acid dipping followed by deionized water rinsing, prior to experiments. In experiments, a brief pre-etch breakthrough step (typically $10 \mathrm{~s}$ at $P_{\text {rf }}=30 \mathrm{~W}$ ) was employed to remove native and/or chemical oxide layers on $\mathrm{Si}$, prior to main etch. The etched depth was measured by stylus profilometry, and the etch rate was then calculated by dividing the etched depth by the etching time (typically $t_{\text {etch }}=2 \mathrm{~min}$ ); moreover, the etched surfaces were examined by using atomic force microscopy (AFM) to measure the rms surface roughness (initially $\sim 0.15 \mathrm{~nm}$ prior to etching), and also by scanning electron microscopy to take the corresponding surface image. Note that no wafer cleaning was done after etching. In addition, 100-nm-thick thermally grown $\mathrm{SiO}_{2}$ films on $\mathrm{Si}$ were also employed in these experiments to measure the etch selectivity of $\mathrm{Si}$ over $\mathrm{SiO}_{2}$.

Several plasma diagnostics were employed to characterize the plasma around the wafer position during processing: optical emission spectroscopy (OES), Fourier transform infraredtransmission absorption spectroscopy (FTIR-TAS), ${ }^{66,68}$ quadrupole mass spectrometry (QMS), and Langmuir probe measurement. Moreover, FTIR-reflection absorption spectroscopy (FTIR-RAS) was also employed to characterize substrate surfaces during processing, in single reflection mode for the IR beam at an incidence angle of $80^{\circ} .68$ The dc self-bias voltage $V_{\mathrm{dc}}$ at the wafer stage was monitored by a voltage probe to give a measure of the ion incident energy $E_{i}=V_{p}-V_{\mathrm{dc}}$ onto substrates, taking into account the plasma potential $V_{p}$ measured by the Langmuir probe.

\section{B. Experimental results and discussion}

\section{Etch rate and rms surface roughness}

Figure 17 shows the etch rates of $\mathrm{Si}$ and $\mathrm{SiO}_{2}$ and etch selectivity of $\mathrm{Si}$ over $\mathrm{SiO}_{2}$, together with the rms surface roughness of $\mathrm{Si}$, as a function of ion incident energy $E_{i}$, experimentally obtained in ICP plasma etching of $\mathrm{Si}$ in $\mathrm{Cl}_{2}$. The error bars in the figure indicate the variation in the raw data for more than ten etching experiments using sample wafers with different as well as similar resistivities and cleaning recipes. Note that in these experiments, the rms roughness was measured for five different scan areas ranging from $0.05 \times 0.05 \mu \mathrm{m}^{2}$ to $1 \times 1 \mu \mathrm{m}^{2}$, showing the known behavior that the larger the scan area, the larger the roughness, ${ }^{93}$ although the $E_{i}$ dependence of the roughness remained unchanged. In practice, the data on the rms roughness shown are those acquired for smaller scan areas of $0.05 \times 0.05 \mu \mathrm{m}^{2}, 0.1 \times 0.1 \mu \mathrm{m}^{2}$, and $0.25 \times 0.25 \mu \mathrm{m}^{2}$ with the resolution of $256 \times 256$ pixels, using AFM in tapping mode with a tip radius less than $10 \mathrm{~nm}$. Also shown in the figure are the ERs and RMSs of Si at $t=120 \mathrm{~s}$, obtained through the ASCeM-3D simulation of $\mathrm{Si}$ etching in $\mathrm{Cl}_{2}$ plasma for different $E_{i}=20-500 \mathrm{eV}$ with $\theta_{i}=0^{\circ}$ (normal ion incidence) as shown in Figs. 5(a) and 6.

The experiments indicate that as the bias power $P_{\mathrm{rf}}$ or ion energy $E_{i}$ is increased, the etch rates of $\mathrm{Si}$ and $\mathrm{SiO}_{2}$ increase and the selectivity of $\mathrm{Si}$ over $\mathrm{SiO}_{2}$ decreases; on the other 


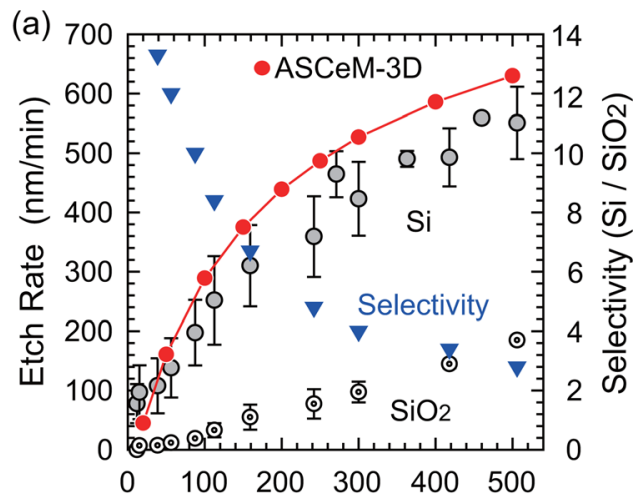

(b)

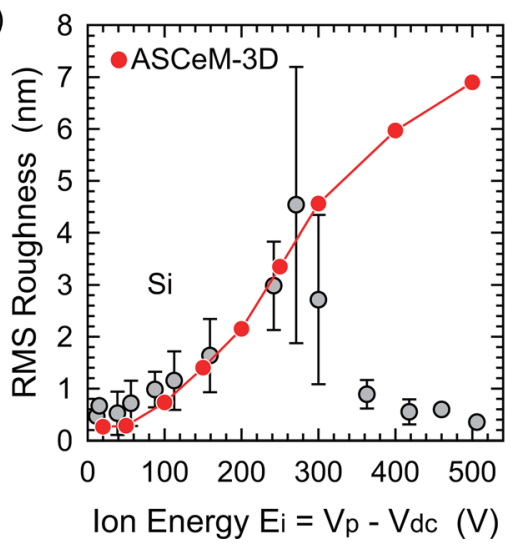

FIG. 17. (Color online) (a) Etch rates of $\mathrm{Si}$ and $\mathrm{SiO}_{2}$ and etch selectivity of $\mathrm{Si}$ over $\mathrm{SiO}_{2}$, and (b) rms surface roughness of $\mathrm{Si}$ as a function of ion incident energy $E_{i}=V_{p}-V_{\mathrm{dc}}$, experimentally obtained in ICP plasma etching of $\mathrm{Si}$ in $\mathrm{Cl}_{2}\left(P_{\mathrm{ICP}}=450 \mathrm{~W}\right.$ at $13.56 \mathrm{MHz}, P_{\mathrm{rf}}=0-200 \mathrm{~W}$ at $13.56 \mathrm{MHz}$, $\left.P_{0}=20 \mathrm{mTorr}, F_{0}=20 \mathrm{sccm}, t_{\mathrm{etch}}=2 \mathrm{~min}\right)$. Here, $V_{p}$ is the plasma potential measured by a Langmuir probe, and $V_{\mathrm{dc}}$ is the dc self-bias voltage at the wafer stage measured by a voltage probe. Also shown are the ERs and RMSs of Si at $t=120 \mathrm{~s}$, obtained through the ASCeM-3D simulation of Si etching in $\mathrm{Cl}_{2}$ plasma for different $E_{i}=20-500 \mathrm{eV}$ with $\theta_{i}=0^{\circ}$ (normal ion incidence) as shown in Figs. 5(a) and 6.

hand, the rms surface roughness of $\mathrm{Si}$ increases, peaks at around $E_{i} \approx 250 \mathrm{eV}$, and then decreases substantially. A comparison between experiments and simulations indicates that the ASCeM-3D reproduces well the increase in $\mathrm{Si}$ etch rate with increasing ion energy in the whole $E_{i}$ range investigated and the increase in $\mathrm{rms}$ roughness of $\mathrm{Si}$ surfaces at $E_{i}<250 \mathrm{eV}$; in contrast, the ASCeM-3D does not reproduce the decrease in rms roughness of $\mathrm{Si}$ at $E_{i}>250 \mathrm{eV}$, where the roughness decreases with $E_{i}$ in experiments, while it continues to increase with $E_{i}$ in simulations. Therefore, it is concluded that the ASCeM-3D can reproduce the evolution of $3 \mathrm{D}$ roughened surface features during plasma etching of $\mathrm{Si}$ at lower $E_{i}<250 \mathrm{eV}$, while the present ASCeM-3D model cannot reproduce it at high $E_{i}>250 \mathrm{eV}$.

The disagreement of the rms surface roughness at high $E_{i}$ between the ASCeM-3D and experiments may be attributable to effects such as chemical etching, surface diffusion (or migration) of atoms, uv radiation, and etch byproducts coming from the plasma; in practice, the former two would tend to smooth surfaces at increased temperatures. ${ }^{94,95}$ The ASCeM-3D simulations of $\mathrm{Si}$ etching in $\mathrm{Cl}_{2}$ plasma indicated that, e.g., at an increased surface temperature $T_{s}=500 \mathrm{~K}$, the rms roughness is reduced substantially to a low level $(\mathrm{RMS} \approx 0.2 \mathrm{~nm})$ and the ripple structures fade away, with the etch rate being increased by about $20 \%$ (as compared to the results at $T_{s}=320 \mathrm{~K}$ in Sec. II B) owing to effects of chemical etching reactions enhanced. Thus, the increased $T_{s}$ at increased $E_{i}$ is considered to be a cause of the disagreement because the surface temperature during plasma etching was found to increase with increasing $P_{\text {rf }}$ or $E_{i}$, being $T_{s} \approx 500 \mathrm{~K}$ at $E_{i}=500 \mathrm{eV}$, estimated through FTIR-RAS measurement calibrated by using temperature labels. ${ }^{96}$

\section{Etch byproducts in the plasma}

Figure 18 shows the peak absorbance of $\mathrm{SiCl}_{4}$ at $620 \mathrm{~cm}^{-1}\left(\nu_{3}\right.$ fundamental vibrational band $\left.{ }^{97}\right)$ as a function of ion incident energy $E_{i}=V_{p}-V_{\mathrm{dc}}$, measured by FTIR-TAS during ICP plasma etching of $\mathrm{Si}$ in $\mathrm{Cl}_{2}$. The experimental conditions are the same as those of Fig. 17, and the calibration was made by filling the ICP plasma chamber with pure $\mathrm{SiCl}_{4}$ gases at different pressures in the range $P_{0}=0.05-50$ mTorr without discharge to estimate the absolute concentration of etch byproduct neutrals $\mathrm{SiCl}_{4}$ in the plasma. ${ }^{6,68}$ The experiments indicate that the peak absorbance of the $620-\mathrm{cm}^{-1} \mathrm{SiCl}_{4}$ band and thus the concentration of $\mathrm{SiCl}_{4}$ in the plasma increases with increasing bias power $P_{\text {rf }}$ or ion energy $E_{i}$, corresponding to the increase in etch rate of Si with $E_{i}$ as shown in Fig. 17(a); in effect, the concentration of $\mathrm{SiCl}_{4}$ or byproduct neutrals in the plasma during etching is more than $10 \%$ of the feedstock $\mathrm{Cl}_{2}$ gas density, $\left[\mathrm{SiCl}_{4}\right] /\left[\mathrm{Cl}_{2}\right]>0.1$, at $E_{i}>200 \mathrm{eV}$. However, as shown in Figs. 11(a) and 12, the ASCeM-3D simulation of Si etching in $\mathrm{Cl}_{2}$ plasma with $\theta_{i}=0^{\circ}$ (normal ion incidence) does not reproduce the decrease in rms surface roughness of $\mathrm{Si}$ in the presence of incoming fluxes of etch/sputter byproducts from the plasma; in effect, the ASCeM-3D indicates that the rms roughness increases with increasing returning probability $P_{r}$ or byproduct flux $\Gamma_{p}^{0} / \Gamma_{i}^{0}$ although the etch rate decreases slightly with increasing $P_{r}$ or $\Gamma_{p}^{0} / \Gamma_{i}^{0}$.

Figure 19 shows the integrated intensities of feedstock gas ions $\mathrm{Cl}_{x}^{+}(x=1,2)$ and etch byproduct ions $\mathrm{SiCl}_{x}^{+}$

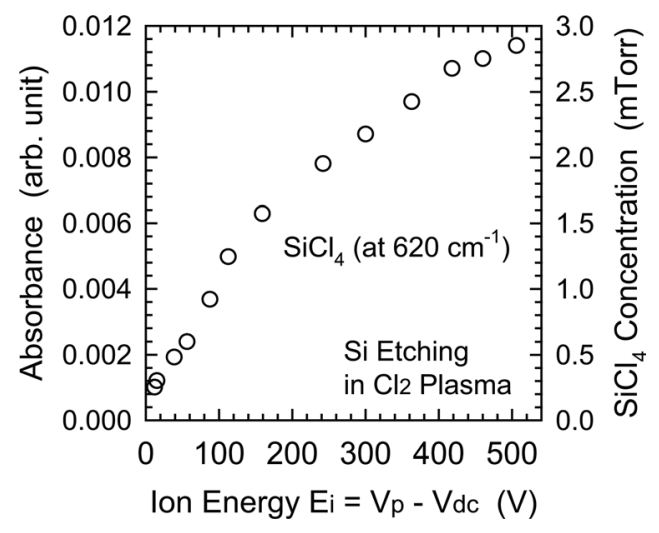

FIG. 18. Peak absorbance of $\mathrm{SiCl}_{4}$ at $620 \mathrm{~cm}^{-1}$ as a function of ion incident energy $E_{i}=V_{p}-V_{\mathrm{dc}}$, measured by FTIR-TAS during ICP plasma etching of $\mathrm{Si}$ in $\mathrm{Cl}_{2}$. The experimental conditions are the same as those of Fig. 17. The calibration was made by filling the plasma chamber with pure $\mathrm{SiCl}_{4}$ gases at different pressures $P_{0}$ without discharge to estimate the absolute concentration of etch byproduct neutrals $\mathrm{SiCl}_{4}$ in the plasma. 


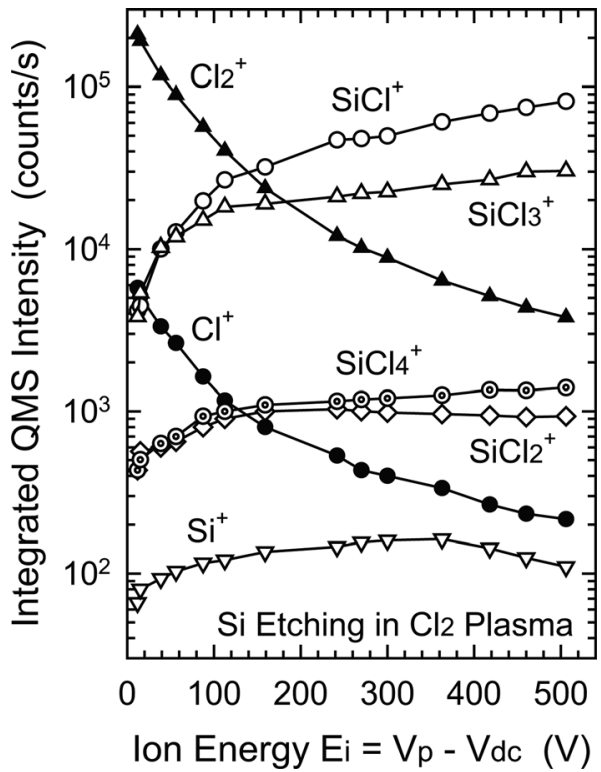

FIG. 19. Integrated intensities of feedstock gas ions $\mathrm{Cl}_{x}^{+}(x=1,2)$ and etch byproduct ions $\mathrm{SiCl}_{x}^{+}(x=0-4)$ as a function of ion incident energy $E_{i}=V_{p}$ - $V_{\mathrm{dc}}$, measured by QMS with the ionizer switched off during ICP plasma etching of $\mathrm{Si}$ in $\mathrm{Cl}_{2}$. The experimental conditions are the same as those of Fig. 17. The QMS signal intensities of isotopic components of the respective ions, originating from naturally abundant isotopes of $\mathrm{Si}\left({ }^{28} \mathrm{Si},{ }^{29} \mathrm{Si},{ }^{30} \mathrm{Si}\right)$ and $\mathrm{Cl}\left({ }^{35} \mathrm{Cl},{ }^{37} \mathrm{Cl}\right)$, were integrated to estimate the amounts of feedstock $\mathrm{Cl}_{x}{ }^{+}$ and byproduct $\mathrm{SiCl}_{x}^{+}$ions in the plasma.

$(x=0-4)$ as a function of ion incident energy $E_{i}=V_{p}-V_{\mathrm{dc}}$, measured by QMS with the ionizer switched off during ICP plasma etching of $\mathrm{Si}$ in $\mathrm{Cl}_{2}$. The experimental conditions are the same as those of Fig. 17, and the QMS signal intensities of isotopic components of the respective ions, originating from naturally abundant isotopes of $\mathrm{Si}\left({ }^{28} \mathrm{Si},{ }^{29} \mathrm{Si},{ }^{30} \mathrm{Si}\right)$ and $\mathrm{Cl}\left({ }^{35} \mathrm{Cl},{ }^{37} \mathrm{Cl}\right)$, were integrated to estimate the amounts of feedstock $\mathrm{Cl}_{x}{ }^{+}$and byproduct $\mathrm{SiCl}_{x}{ }^{+}$ions in the plasma. The experiments indicate that the amounts of feedstock ions $\mathrm{Cl}_{x}{ }^{+}$ in the plasma decrease with increasing bias power $P_{\mathrm{rf}}$ or ion energy $E_{i}$, while those of byproduct ions $\mathrm{SiCl}_{x}{ }^{+}$increase with increasing $P_{\mathrm{rf}}$ or $E_{i}$, corresponding to the increase in etch rate of Si with $E_{i}$ as shown in Fig. 17(a); in effect, at $E_{i}>200 \mathrm{eV}$, the byproduct ions $\mathrm{SiCl}^{+}$and $\mathrm{SiCl}_{3}{ }^{+}$in the plasma during etching are more abundant than the feedstock one $\mathrm{Cl}_{2}{ }^{+},\left[\mathrm{SiCl}_{x}{ }^{+}\right] /\left[\mathrm{Cl}_{2}{ }^{+}\right]>1(x=1,3)$, and so the incoming ion flux from the plasma onto substrate surfaces tends to be dominated by byproduct ions. However, the present ASCeM-3D model cannot simulate the etch-byproduct ion incidence on substrate surfaces because the situation is contradictory to the basic assumption of the ASCeM (as mentioned in Sec. II A 1) that the ASCeM cell is rigid and contains one Si atom therein.

Figure 20 shows typical side views of the $\mathrm{Si}(100)$ surface at after 2000 impacts of $\mathrm{Cl}^{+}$and $\mathrm{SiCl}^{+}$beam ions thereon, together with the depth profiles of $\mathrm{Cl}$ and $\mathrm{Si}$ atoms therein, obtained through the classical MD simulation of etching for $\mathrm{Si} / \mathrm{Cl}$ and $\mathrm{Si} / \mathrm{SiCl}$ systems with different beam energies of $E_{i}=20-500 \mathrm{eV}$ with $\theta_{i}=0^{\circ}$ (normal incidence). Inset is the coverage or areal density of $\mathrm{Cl}$ atoms adsorbed on surfaces, where the simulation cell is a square $32.58 \AA$ on a side with an initial depth of $26 \AA$, and a monolayer (ML) contains 72 $\mathrm{Si}$ atoms $\left(=6.78 \times 10^{14}\right.$ atoms $\left./ \mathrm{cm}^{2}\right)$; in practice, the simulation cell initially contains substrate or target atoms of $20 \mathrm{ML}$

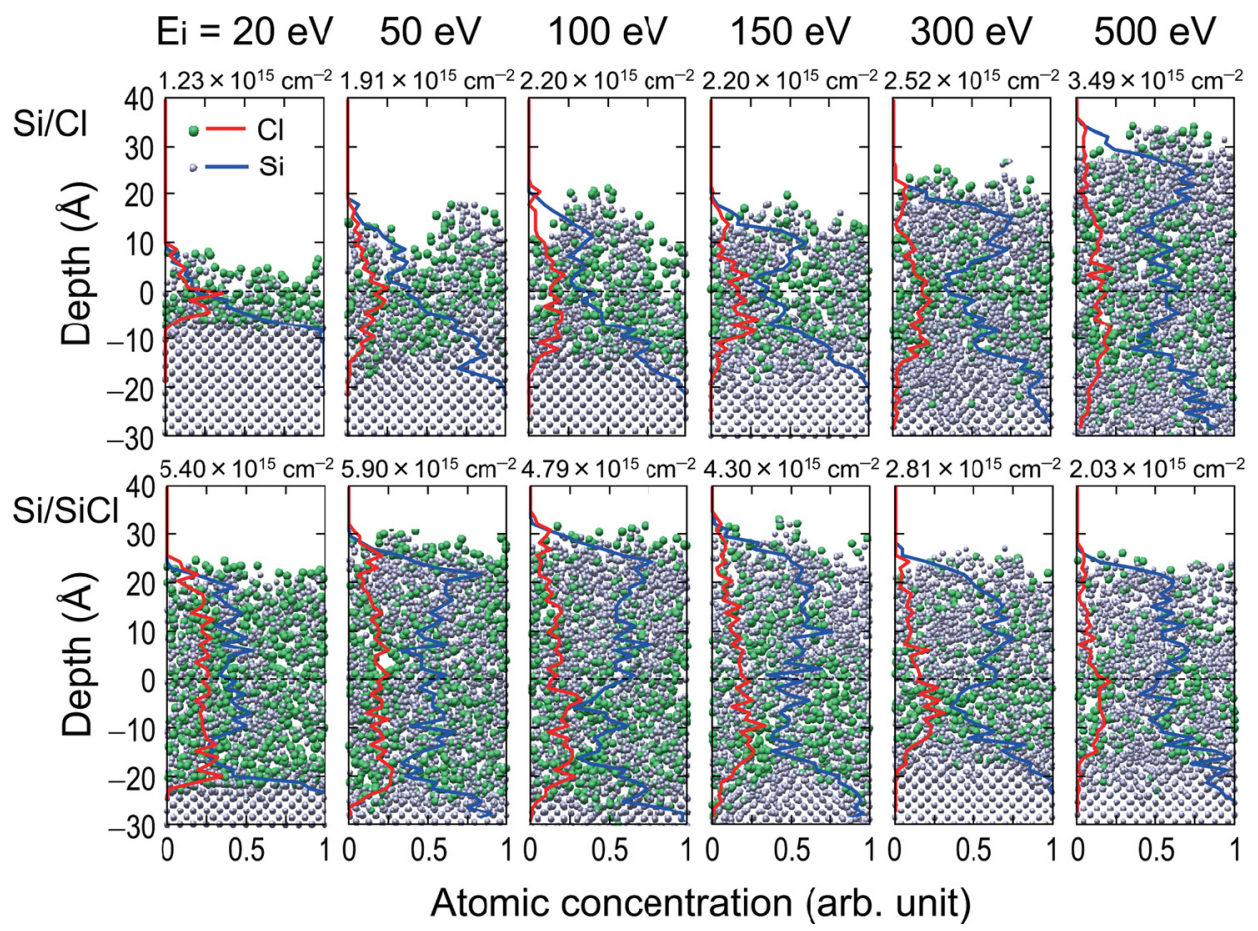

FIg. 20. (Color online) Typical side views of the $\mathrm{Si}(100)$ surface, together with the depth profiles of $\mathrm{Cl}$ and $\mathrm{Si}$ atoms therein, obtained through the classical $\mathrm{MD}$ simulation of $\mathrm{Cl}^{+}$and $\mathrm{SiCl}^{+}$ion incidences with different energies of $E_{i}=20-500 \mathrm{eV}$ at $\theta_{i}=0^{\circ}$ (normal incidence). Note that these are snapshots at after 2000 ion impacts, and the ions are assumed to be charge-neutral species with high translational energies, similarly to the ASCeM model. Inset is the coverage or areal density of $\mathrm{Cl}$ atoms adsorbed on surfaces, where the simulation cell is a square $32.58 \AA$ on a side with an initial depth of $26 \AA$. 
or $1440 \mathrm{Si}$ atoms, which are maintained during etching by sometimes adding a layer of $\mathrm{Si}$ atoms at the bottom. Target atoms in the bottom layer are fixed during the simulation, while periodical boundaries are imposed in the horizontal direction. Note that impinging particles in MD are also assumed to be charge-neutral species with high translational energies, similarly to the ASCeM model, and the interatomic potential employed is an improved Stillinger-Weber type previously developed. ${ }^{98,99}$ The MD simulations indicate that for the $\mathrm{Si} / \mathrm{Cl}$ system, the thickness of surface $\mathrm{SiCl}_{x}$ layers or the penetration depth of $\mathrm{Cl}$ atoms increases with increasing beam energy $E_{i}$, together with the coverage of $\mathrm{Cl}$ atoms adsorbed; on the other hand, for the $\mathrm{Si} / \mathrm{SiCl}$ system, the thickness of $\mathrm{SiCl}_{x}$ layers peaks at $E_{i} \approx 100-150 \mathrm{eV}$, while the coverage of $\mathrm{Cl}$ atoms decreases with increasing $E_{i}$.

Figure 21 shows the effective $\mathrm{Si}$ yield $Y^{*}$ per ion impact as a function of incident energy $E_{i}$, obtained through the MD simulation of $\mathrm{Cl}_{x}^{+}(x=1,2)$ and $\mathrm{SiCl}_{x}{ }^{+}(x=0-4)$ ion incidences on $\operatorname{Si}(100)$ surfaces with different $E_{i}=20-500 \mathrm{eV}$ at $\theta_{i}=0^{\circ}$ (normal incidence) as shown in Fig. 20. Note that in the case of $\mathrm{Cl}_{x}{ }^{+}$incidence, the effective Si yield $Y^{*}$ (per ion) equals the Si yield $Y$ (per ion), which is defined as the total number of $\mathrm{Si}$ atoms desorbed from substrate surfaces per ion impact; on the other hand, the effective yield is $Y^{*}=Y-1$ in the case of $\mathrm{SiCl}_{x}{ }^{+}$incidence, because one $\mathrm{Si}$ atom is incident on surfaces per $\mathrm{SiCl}_{x}^{+}$impact. Thus, the positive $Y^{*}$ indicates the etching, while the negative $Y^{*}$ indicates the deposition. ${ }^{100}$ The results indicate that in $\mathrm{Cl}^{+}, \mathrm{Cl}_{2}{ }^{+}, \mathrm{SiCl}_{3}{ }^{+}$, and $\mathrm{SiCl}_{4}{ }^{+}$incidences, the etching occurs in the whole $E_{i}$ range investigated, where the yield $Y^{*}$ increases with increasing $E_{i}$. On the other hand, in $\mathrm{SiCl}^{+}$and $\mathrm{SiCl}_{2}{ }^{+}$incidences, a transition from deposition to etching occurs at

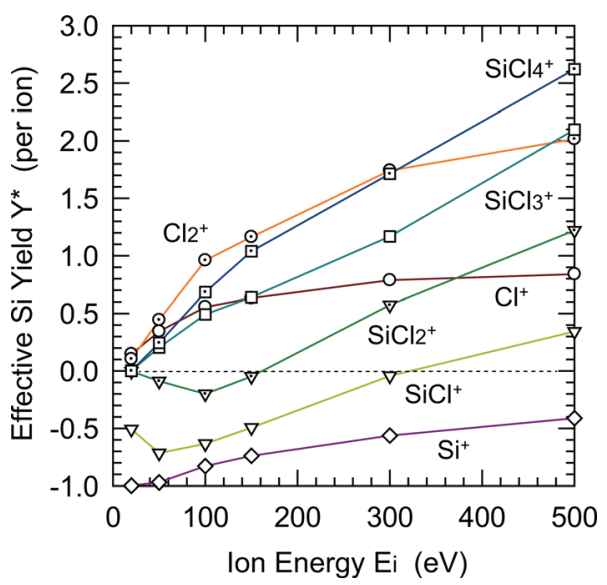

FIG. 21. (Color online) Effective Si yield $Y^{*}$ per ion impact as a function of incident energy $E_{i}$, obtained through the MD simulation of $\mathrm{Cl}_{x}{ }^{+}(x=1,2)$ and $\mathrm{SiCl}_{x}^{+}(x=0-4)$ ion incidences on $\mathrm{Si}(100)$ surfaces with different $E_{i}=20-500 \mathrm{eV}$ at $\theta_{i}=0^{\circ}$ (normal incidence) as shown in Fig. 20. [Reproduced in part with permission from N. Nakazaki, Y. Takao, K. Eriguchi, and K. Ono, Jpn. J. Appl. Phys. 53, 056201 (2014). Copyright 2014 The Japan Society of Applied Physics.] Note that in the case of $\mathrm{Cl}_{x}{ }^{+}$ incidence, the effective Si yield $Y^{*}$ (per ion) equals the Si yield $Y$ (per ion), which is defined as the total number of $\mathrm{Si}$ atoms desorbed from substrate surfaces per ion impact; on the other hand, the effective yield is $Y^{*}=Y-1$ in the case of $\mathrm{SiCl}_{x}{ }^{+}$incidence, because one $\mathrm{Si}$ atom is incident on surfaces per $\mathrm{SiCl}_{x}{ }^{+}$impact. Thus, the positive $Y^{*}$ indicates the etching, while the negative $Y^{*}$ indicates the deposition. around $E_{i} \approx 300$ and $150 \mathrm{eV}$, respectively, where the yield $Y^{*}$ increases with increasing $E_{i}$; moreover, in $\mathrm{Si}^{+}$incidence, the deposition occurs in the whole $E_{i}$ range investigated, where the absolute value of the yield $Y^{*}$ decreases or the deposition is reduced with increasing $E_{i}$. These are consistent with the results of beam experiments previously reported: $\mathrm{SiCl}_{x}^{+}(x=1-3)$ beams of $E_{i}=30 \mathrm{eV}$ (Ref. 101) and $\mathrm{Cl}^{+}$and $\mathrm{SiCl}_{x}^{+}(x=0,1,3)$ beams of $E_{i}=100-500 \mathrm{eV}$ (Ref. 102) incident on Si surfaces.

Similar situations may occur in the present ICP plasma etching of $\mathrm{Si}$ in $\mathrm{Cl}_{2}$ at increased bias powers $P_{\text {rf }}$ or ion energies $E_{i}$; in practice, the experiments exhibit that the amount of etch byproduct ions $\mathrm{SiCl}^{+}$and $\mathrm{SiCl}_{3}{ }^{+}$tends to be larger than that of the feedstock gas ion $\mathrm{Cl}_{2}{ }^{+}$at $E_{i}>200 \mathrm{eV}$, as seen in Fig. 19, owing to increased etch rates. The MD simulations above exhibit that the $\mathrm{SiCl}^{+}$incidence on $\mathrm{Si}$ leads to the deposition at $E_{i}<300 \mathrm{eV}$, while the etching at $E_{i}>300 \mathrm{eV}$, where competitive effects of etching and deposition would play some role in smoothing surfaces at increased $E_{i}$. The results of a previous MD simulation implied that the reflection or scattering behavior of energetic silicon-halogen compounds on $\mathrm{Si}$ is different from that of halogen. ${ }^{103,104}$ Moreover, the surface roughness is known to be reduced by using cluster ion beams, ${ }^{105,106}$ owing to low translational energies of the respective constituent atoms of cluster ions incident on the surface. Thus, the increased effects of etch byproduct molecular ions coming from the plasma at increased $E_{i}$ are considered to be a second cause of the disagreement of the rms roughness at high $E_{i}>250 \mathrm{eV}$ between the ASCeM-3D and experiments as seen in Fig. 17(b). However, a more relevant understanding of the mechanisms for the decrease in roughness at increased $E_{i}$ presently observed in experiments will require a further study including MD simulations of oblique incidence of byproduct ions $\mathrm{SiCl}_{x}^{+}$on $\mathrm{Si}$ to reveal their reflection characteristics thereon, which is crucial for surface roughening as well as rippling as mentioned in Sec. II B 7.

\section{CONCLUSIONS}

Atomic- or nanometer-scale surface roughness and ripples formed during $\mathrm{Si}$ etching in $\mathrm{Cl}_{2}$ and $\mathrm{Cl}_{2} / \mathrm{O}_{2}$ plasmas have been investigated by developing an ASCeM-3D model for plasma- surface interactions and the feature profile evolution during plasma etching. The ASCeM-3D is a 3D Monte Carlo-based simulation model, taking into account the behavior of energetic ions $\mathrm{Cl}^{+}$, neutral reactants or etchants $\mathrm{Cl}$, reactive neutrals $\mathrm{O}$, and etch products and byproducts of $\mathrm{SiCl}_{x}$ and $\mathrm{SiCl}_{x} \mathrm{O}_{y}$ in microstructures, and on feature surfaces thereon. The surface chemistry and kinetics included surface chlorination, chemical etching, ion-enhanced etching, sputtering, surface oxidation, redeposition of etch products desorbed from feature surfaces being etched, and deposition of etch byproducts coming from the plasma, where the latter three are responsible for passivation layer formation on feature surfaces. The model also took into account two-body elastic collisions between energetic ions and substrate $\mathrm{Si}$ atoms, to analyze the ion reflection or scattering from feature 
surfaces on incidence and/or the ion penetration into substrates, along with geometrical shadowing of the feature and surface reemission of neutrals. The simulation domain was taken to consist of small atomic-size cubic cells $2.7 \AA$ on a side, and the evolving interfaces were represented by removing $\mathrm{Si}$ atoms from and/or allocating them to the cells concerned.

Calculations were performed for a square substrate $50 \mathrm{~nm}$ on a side by varying the ion incidence angle onto substrate surfaces, typically with an incoming ion energy, ion flux, and neutral reactant-to-ion flux ratio of $E_{i}=100 \mathrm{eV}$, $\Gamma_{i}^{0}=1.0 \times 10^{16} \mathrm{~cm}^{-2} \mathrm{~s}^{-1}$, and $\Gamma_{n}^{0} / \Gamma_{i}^{0}=100$ in the absence of incoming fluxes of oxygen and etch byproducts $\left(\Gamma_{o}{ }^{0}=\Gamma_{p}{ }^{0}=0\right)$. Numerical results showed that nanoscale roughened surface features evolve with time during etching, depending markedly on the angle $\theta_{i}$ of ion incidence; in effect, at $\theta_{i}=0^{\circ}$ or normal incidence, concavo-convex features are formed randomly on surfaces. On the other hand, at increased $\theta_{i}=45^{\circ}$ or oblique incidence, ripple structures with a wavelength of the order of $15 \mathrm{~nm}$ are formed on surfaces perpendicularly to the direction of ion incidence; in contrast, at further increased $\theta_{i} \geq 75^{\circ}$ or grazing incidence, small ripples or slitlike grooves with a wavelength of $<5 \mathrm{~nm}$ are formed on surfaces parallel to the direction of ion incidence. In addition, it was found that at $\theta_{i} \geq 75^{\circ}$, some of them tend to enlarge significantly with time to form a wide, deep groove $(>10 \mathrm{~nm}$ in width and $>30 \mathrm{~nm}$ in depth) on etched surfaces, which appeared at some simulations and did not appear at others, being attributable presumably to the Monte Carlo calculation of stochastic processes using random numbers.

Such surface roughening and rippling in response to ion incidence angle onto substrate surfaces were also found to depend significantly on ion energy $\left(E_{i}=20-500 \mathrm{eV}\right)$ and incoming fluxes of neutral reactants $\left(\Gamma_{n}^{0} / \Gamma_{i}^{0}=10-500\right)$, oxygen $\left(\Gamma_{o}^{0} / \Gamma_{i}^{0}=0-0.5\right)$, and etch byproducts $\left(P_{r}=0-0.5\right.$ or $\left.\Gamma_{p}^{0} / \Gamma_{i}^{0} \approx 0-1.0\right)$ : the increase in $E_{i}$ tends to enhance the roughening and rippling. The increase in $\Gamma_{n}{ }^{0}$ tends to suppress the roughening at $\theta_{i}=0^{\circ}$ (owing to increased chemical effects of chemical and ion-enhanced etching reactions), while it tends to enhance the rippling at $\theta_{i} \geq 45^{\circ}$. The increase in $\Gamma_{o}{ }^{0}$ and $\Gamma_{p}{ }^{0}$ (or the passivation layer formation) tends to increase the roughness at $\theta_{i}=0^{\circ}$ (owing to local micromasking caused by local surface oxidation and byproduct deposition), while it tends to suppress the rippling at $\theta_{i} \geq 45^{\circ}$. The 2D-PSD analysis of the roughened feature surfaces simulated was made in some cases to further characterize the lateral as well as vertical extent of the roughness. Then, possible mechanisms responsible for the formation and evolution of surface roughness and ripples during plasma etching have been discussed, including stochastic roughening, local micromasking, and effects of ion reflection, surface temperature, and ion angular distribution. The ASCeM-3D implied that effects of the ion reflection from roughened feature surfaces on incidence is crucial for the evolution of surface roughening and rippling during plasma etching, while the increased surface temperature (or chemical etching) and the increased ion angular distribution (or ion temperature) cause smoothing surfaces.
Moreover, plasma etching experiments of blank Si substrates in $\mathrm{Cl}_{2}$ were conducted by varying the $\mathrm{rf}$ bias power or ion incident energy to verify the validity of our ASCeM-3D model. A comparison of the etch rate and rms surface roughness between experiments and simulations indicated that the ASCeM-3D with $\theta_{i}=0^{\circ}$ reproduces well the experiments at $E_{i}<250 \mathrm{eV}$; on the other hand, the ASCeM-3D does not reproduce the rms roughness at high $E_{i}>250 \mathrm{eV}$, although it reproduces well the etch rate behavior thereat, where the roughness decreases with increasing $E_{i}$ in experiments, while it continues to increase with $E_{i}$ in simulations. Possible mechanisms for this disagreement between the ASCeM-3D and experiments have been discussed with the help of several plasma and surface diagnostics and classical MD simulations for $\mathrm{Si} / \mathrm{Cl}$ and $\mathrm{Si} / \mathrm{SiCl}$ systems, being attributable to effects of the increased incoming fluxes of etch byproduct ions $\mathrm{SiCl}_{x}{ }^{+}$and/or increased surface temperatures at increased $E_{i}$.

A more relevant understanding of the mechanisms for the surface rippling during plasma etching presently observed in ASCeM-3D simulations $\left(\theta_{i} \geq 45^{\circ}\right)$ will require a further study including experimental demonstrations of it, where some control of the plasma sheath on blank Si substrate surfaces will be indispensable for off-normal or oblique ion incidence thereon. A more relevant understanding of the mechanisms for the decrease in roughness at increased $E_{i}$ presently observed in plasma etching experiments $\left(\theta_{i}=0^{\circ}\right)$ will require a further study including $\mathrm{MD}$ simulations of oblique incidence of byproduct ions $\mathrm{SiCl}_{x}{ }^{+}$on $\mathrm{Si}$ to reveal their reflection behavior thereon. Further studies are also now in progress for a more comprehensive understanding of the mechanisms responsible for the evolution of surface roughness and ripples during plasma etching; in particular, several plasma etching experiments of Si previously reported $\left(\theta_{i}=0^{\circ}\right)$ showed a continuous increase in roughness with time $>10-20 \mathrm{~min}$, which cannot be fully reproduced by the present ASCeM-3D.

The surface roughening and rippling during plasma etching, as observed in our ASCeM-3D simulations, could occur on feature sidewalls and bottom surfaces in plasma etching for pattern definition. Thus, such numerical investigations followed by a comparison with experiments would be useful and indispensable to find plasma etching conditions for controlling the surface roughness and ripples in the fabrication of nanoscale microelectronic devices. The surface rippling in response to ion incidence angle on surfaces in plasma etching environments would also be useful for the fabrication of well-organized nanostructures using plasmas, as well as be of interest from the viewpoint of a fundamental study of plasma-surface interactions, we believe.

\section{ACKNOWLEDGMENTS}

This work was supported by a Grant-in-Aid for Scientific Research on Innovative Areas (Frontier science of interactions between plasmas and nanointerfaces) from the Ministry of Education, Culture, Sports, Science and Technology, Japan. One of the authors (H.T.) was supported by Research 
Fellowships of the Japan Society for the Promotion of Science for Young Scientists. The authors would also like to thank T. Hatsuse for assistance with ASCeM-3D numerical simulations.

${ }^{1}$ H. Abe, M. Yoneda, and N. Fujiwara, Jpn. J. Appl. Phys. 47, 1435 (2008).

${ }^{2}$ V. M. Donnelly and A. Kornblit, J. Vac. Sci. Technol. A 31, 050825 (2013).

${ }^{3}$ B. Wu, A. Kumar, and S. Pamarthy, J. Appl. Phys. 108, 051101 (2010).

${ }^{4}$ See http://www.itrs.net for International Technology Roadmap for Semiconductors (ITRS) 2013 Edition.

${ }^{5}$ K. J. Kanarik, G. Kamarthy, and R. A. Gottscho, Solid State Technol. 55, 15 (2012).

${ }^{6}$ E. Pargon, M. Darnon, O. Joubert, T. Chevolleau, L. Vallier, L. Mollard, and T. Lill, J. Vac. Sci. Technol. B 23, 1913 (2005).

${ }^{7}$ P. Pan and L. Liu, J. Vac. Sci. Technol. B 15, 1752 (1997).

${ }^{8}$ D. J. Thomas, P. Southworth, M. C. Flowers, and R. Greef, J. Vac. Sci. Technol. B 7, 1325 (1989).

${ }^{9}$ R. Pétri, P. Brault, O. Vatel, D. Henry, E. André, P. Dumas, and F. Salvan, J. Appl. Phys. 75, 7498 (1994).

${ }^{10}$ H. Feil, J. Dieleman, and B. J. Garrison, J. Appl. Phys. 74, 1303 (1993).

${ }^{11}$ K.-T. Sung and S. W. Pang, Jpn. J. Appl. Phys., Part 1 33, 7112 (1994).

${ }^{12}$ M. J. M. Vugts, M. F. A. Eurlings, L. J. F. Hermans, and H. C. W. Beijerinck, J. Vac. Sci. Technol. A 14, 2780 (1996).

${ }^{13}$ P. Brault, P. Dumas, and F. Salvan, J. Phys. Condens. Matter 10, L27 (1998).

${ }^{14}$ Y.-P. Zhao, J. T. Drotar, G.-C. Wang, and T.-M. Lu, Phys. Rev. Lett. 82, 4882 (1999).

${ }^{15}$ J. T. Drotar, Y.-P. Zhao, T.-M. Lu, and G.-C. Wang, Phys. Rev. B 61, 3012 (2000).

${ }^{16}$ J. T. Drotar, Y.-P. Zhao, T.-M. Lu, and G.-C. Wang, Phys. Rev. B 62, 2118 (2000).

${ }^{17}$ E. Gogolides, C. Boukouras, G. Kokkoris, O. Brani, A. Tserepi, and V. Constantoudis, Microelectron. Eng. 73-74, 312 (2004).

${ }^{18}$ A. A. E. Stevens and H. C. W. Beijerinck, J. Vac. Sci. Technol. A 23, 126 (2005).

${ }^{19}$ X. Hua, S. Engelmann, G. S. Oehrlein, P. Jiang, P. Lazzeri, E. Iacob, and M. Anderle, J. Vac. Sci. Technol. B 24, 1850 (2006).

${ }^{20}$ Y. Yin, S. Rasgon, and H. H. Sawin, J. Vac. Sci. Technol. B 24, 2360 (2006).

${ }^{21}$ Y. Yin and H. H. Sawin, J. Vac. Sci. Technol. A 25, 802 (2007).

${ }^{22}$ E. Zakka, V. Constantoudis, and E. Gogolides, IEEE Trans. Plasma Sci. 35, 1359 (2007).

${ }^{23}$ G. Kokkoris, V. Constantoudis, P. Angelikopoulos, G. Boulousis, and E. Gogolides, Phys. Rev. B 76, 193405 (2007).

${ }^{24}$ Y. Yin and H. H. Sawin, J. Vac. Sci. Technol. A 26, 151 (2008).

${ }^{25}$ W. S. Hwang, B.-J. Cho, D. S. H. Chan, S. W. Lee, and W. J. Yoo, J. Electrochem. Soc. 155, H6 (2008).

${ }^{26}$ M. Martina and G. Cunge, J. Vac. Sci. Technol. B 26, 1281 (2008).

${ }^{27}$ A. R. Pal, R. L. Bruce, F. Weilnboeck, S. Engelmann, T. Lin, M.-S. Kuo, R. Phaneuf, and G. S. Oehrlein1, J. Appl. Phys. 105, 013311 (2009).

${ }^{28}$ W. Guo and H. H. Sawin, J. Phys. D: Appl. Phys. 42, 194014 (2009).

${ }^{29}$ W. Guo and H. H. Sawin, J. Vac. Sci. Technol. A 28, 259 (2010).

${ }^{30}$ J. J. Végh and D. B. Graves, Plasma Sources Sci. Technol. 19, 045005 (2010).

${ }^{31}$ E. Gogolides, V. Constantoudis, G. Kokkoris, D. Kontziampasis, K. Tsougeni, G. Boulousis, M. Vlachopoulou, and A. Tserepi, J. Phys. D: Appl. Phys. 44, 174021 (2011).

${ }^{32}$ J. Thiault, J. Foucher, J. H. Tortai, O. Joubert, S. Ladis, and S. Pauliac, J. Vac. Sci. Technol. B 23, 3075 (2005).

${ }^{33}$ E. Gogolides, V. Constantoudis, G. P. Patsis, and A. Tserepi, Microelectron. Eng. 83, 1067 (2006).

${ }^{34}$ K. Patel, T.-J. King Liu, and C. J. Spanos, IEEE Trans. Electron Devices 56, 3055 (2009).

${ }^{35}$ X. Sun and T.-J. King Liu, IEEE Trans. Semicond. Manuf. 23, 311 (2010).

${ }^{36}$ E. Altamirano-Sánchez, V. Paraschiv, M. Demand, and W. Boullart, Microelectron. Eng. 88, 2871 (2011).
${ }^{37}$ K. Eriguchi, Y. Takao, and K. Ono, J. Vac. Sci. Technol. A 29, 041303 (2011).

${ }^{38}$ K. S. Chen, A. Ayon, and S. M. Spearing, J. Am. Ceram. Soc. 83, 1476 (2000).

${ }^{39}$ N. Tayebi and A. A. Polycarpou, J. Appl. Phys. 98, 073528 (2005).

${ }^{40}$ Y. Osano and K. Ono, Jpn. J. Appl. Phys., Part 1 44, 8650 (2005).

${ }^{41}$ Y. Osano M. Mori, N. Itabashi, K. Takahashi, K. Eriguchi, and K. Ono, Jpn. J. Appl. Phys., Part 1 45, 8157 (2006).

${ }^{42}$ Y. Osano and K. Ono, J. Vac. Sci. Technol. B 26, 1425 (2008).

${ }^{43}$ K. Ono, H. Ohta, and K. Eriguchi, Thin Solid Films, 518, 3461 (2010).

${ }^{44}$ H. Tsuda, M. Mori, Y. Takao, K. Eriguchi, and K. Ono, Thin Solid Films 518, 3475 (2010).

${ }^{45}$ H. Tsuda, M. Mori, Y. Takao, K. Eriguchi, and K. Ono, Jpn. J. Appl. Phys. 49, 08JE01 (2010).

${ }^{46}$ H. Tsuda, H. Miyata, Y. Takao, K. Eriguchi, and K. Ono, Jpn. J. Appl. Phys. 50, 08JE06 (2011).

${ }^{47}$ H. Tsuda, Y. Takao, K. Eriguchi, and K. Ono, Jpn. J. Appl. Phys. 51, 08HC01 (2012).

${ }^{48}$ A. P. Mahorowala and H. H. Sawin, J. Vac. Sci. Technol. B 20, 1064 (2002).

${ }^{49}$ E. A. Ogryzlo, D. E. Ibbotson, D. L. Flamm, and J. A. Mucha, J. Appl. Phys. 67, 3115 (1990).

${ }^{50}$ W. Guo and H. H. Sawin, J. Vac. Sci. Technol. A 27, 1326 (2009).

${ }^{51}$ W. Guo, B. Bai, and H. H. Sawin, J. Vac. Sci. Technol. A 27, 388 (2009).

${ }^{52}$ W. Jin, S. A. Vitale, and H. H. Sawin, J. Vac. Sci. Technol. A 20, 2106 (2002).

${ }^{53}$ K. Wittmaack, Phys. Rev. B 68, 235211 (2003).

${ }^{54}$ J. P. Chang and H. H. Sawin, J. Vac. Sci. Technol. A 15, 610 (1997).

${ }^{55}$ S. Tachi, K. Tsujimoto, S. Arai, and T. Kure, J. Vac. Sci. Technol. A 9, 796 (1991).

${ }^{56}$ J. P. Chang and H. H. Sawin, J. Vac. Sci. Technol. B 19, 1319 (2001).

${ }^{57}$ D. J. Oostra, R. P. van Ingen, A. Haring, A. E. de Vries, and G. N. A. Van Veen, Appl. Phys. Lett. 50, 1506 (1987).

${ }^{58}$ R. N. Tait, T. Smy, and M. J. Brett, Thin Solid Films 187, 375 (1990).

${ }^{59} \mathrm{P}$. Sigmund, in Sputtering by Particle Bombardment I. Physical Sputtering of Single-Element Solids, edited by R. Behrisch (Springer, Berlin, 1981), Chap. 2, pp. 9-71.

${ }^{60}$ J. P. Biersack and W. Eckstein, Appl. Phys. A 34, 73 (1984).

${ }^{61}$ J. F. Ziegler, J. Biersack, and U. Littmark, The Stopping and Range of Ions in Matter, edited by J. F. Ziegler (Pergamon, New York, 1985), Chap. 2, pp. 14-65.

${ }^{62}$ J. F. Ziegler, J. P. Biersack, and M. D. Ziegler, SRIM-The Stopping and Range of Ions in Matter (SRIM, Chester, MD, 2008), Chap. 2, pp. 2-1-49.

${ }^{63}$ J. Hoshen and R. Kopelman, Phys. Rev. B 14, 3438 (1976).

${ }^{64}$ J. Hoshen, R. Kopelman, and E. M. Monberg, J. Stat. Phys. 19, 219 (1978).

${ }^{65}$ J. Hoshen, P. Klymko, and R. Kopelman, J. Stat. Phys. 21, 583 (1979).

${ }^{66}$ K. Ono, M. Tuda, K. Nishikawa, T. Oomori, and K. Namba, Jpn. J. Appl. Phys., Part 1 33, 4424 (1994).

${ }^{67}$ M. Tuda and K. Ono, Jpn. J. Appl. Phys., Part 1 36, 2482 (1997).

${ }^{68}$ K. Nishikawa, T. Oomori, and K. Ono, J. Vac. Sci. Technol. B 17, 127 (1999).

${ }^{69}$ A.-D. Brown, J. Erlebacher, W.-L. Chan, and E. Chanson, Phys. Rev. Lett. 95, 056101 (2005).

${ }^{70}$ A.-D. Brown and J. Erlebacher, Phys. Rev. B 72, 075350 (2005).

${ }^{71}$ B. Ziberi, F. Frost, Th. Höche, and B. Rauschenbach, Phys. Rev. B 72, 235310 (2005).

${ }^{72}$ W. L. Chan and E. Chason, J. Appl. Phys. 101, 121301 (2007).

${ }^{73}$ B. Ziberi, F. Frost, M. Tartz, H. Neumann, and B. Rauschenbach, Appl. Phys. Lett. 92, 063102 (2008).

${ }^{74}$ B. Ziberi, M. Cornejo, F. Frost, and B. Rauschenbach, J. Phys.: Condens. Matter 21, 224003 (2009).

${ }^{75}$ T. K. Chini, D. P. Datta, and S. R. Bhattacharyya, J. Phys.: Condens. Matter 21, 224004 (2009).

${ }^{76}$ S. A. Pahlovy, S. F. Mahmud, K. Yanagimoto, and I. Miyamoto, J. Vac. Sci. Technol. A 29, 021015 (2011).

${ }^{77}$ R. M. Bradley and J. M. E. Harper, J. Vac. Sci. Technol. A 6, 2390 (1988).

${ }^{78}$ R. Cuerno, H. A. Makse, S. Tomassone, S. T. Harrington, and H. E. Stanley, Phys. Rev. Lett. 75, 4464 (1995).

${ }^{79}$ I. Koponen, M. Hautala, and O.-P. Sievänen, Phys. Rev. Lett. 78, 2612 (1997).

${ }^{80}$ G. Carter, J. Appl. Phys. 85, 455 (1999).

${ }^{81}$ M. A. Makeev and A.-L. Barabási, Appl. Phys. Lett. 71, 2800 (1997). 
${ }^{82}$ M. A. Makeev, R. Cuerno, and A.-L. Barabási, Nucl. Instrum. Methods B 197, 185 (2002).

${ }^{83}$ J. Muñoz-García, M. Castro, and R. Cuerno, Phys. Rev. Lett. 96, 086101 (2006).

${ }^{84}$ T. Morimoto, Jpn. J. Appl. Phys., Part 1 32, 1253 (1993).

${ }^{85}$ G. S. Oehrlein, J. F. Rembetski, and E. H. Payne, J. Vac. Sci. Technol. B 8, 1199 (1990).

${ }^{86}$ T. Hayakawa, T. Suzuki, T. Uesugi, and Y. Mitsushima, Jpn. J. Appl. Phys., Part 1 37, 5 (1998).

${ }^{87} \mathrm{~K}$. Nakashima, Y. Watanabe, T. Yoshida, and Y. Mitsushima, J. Electrochem. Soc. 147, 4294 (2000).

${ }^{88}$ R. Dussart, X. Mellhaoui, T. Tillocher, P. Lefaucheux, M. Volatier, C. SocquetClerc, P. Brault, and P. Ranson, J. Phys. D: Appl. Phys. 38, 3395 (2005).

${ }^{89}$ J. K. Lawson, D. M. Aikens, R. E. English, Jr., and C. R. Wolfe, Proc. SPIE 2775, 345 (1996).

${ }^{90}$ A. Duparré, J. Ferre-Borrull, S. Gliech, G. Notni, J. Steinert, and J. M. Bennett, Appl. Opt. 41, 154 (2002).

${ }^{91}$ V. V. Yashchuk, A. D. Franck, S. C. Irick, M. R. Howells, A. A. MacDowell, and W. R. McKinney, Proc. SPIE 5858, 58580A (2005).

${ }^{92}$ M. Senthilkumar, N. K. Sahoo, S. Thakur, and R. B. Tokas, Appl. Surf. Sci. 252, 1608 (2005).

${ }^{93}$ K. Boussu, B. Van der Bruggen, A. Volodin, J. Snauwaert, C. Van Haesendonck, and C. Vandecasttele, J. Colloid Interface Sci. 286, 632 (2005).
${ }^{94}$ V. S. Aliev and V. N. Kruchinin, Surf. Sci. 442, 206 (1999).

${ }^{95}$ Y.-W. Mo, J. Kleiner, M. B. Webb, and M. G. Lagally, Surf. Sci. 268, 275 (1992).

${ }^{96}$ N. Nakazaki, H. Tsuda, Y. Takao, K. Eriguchi, and K. Ono (submitted).

${ }^{97} \mathrm{~K}$. Nakamoto, Infrared and Raman Spectra of Inorganic and Coordination Compounds, 5th ed., Part A: Theory and Applications in Inorganic Chemistry (Wiley, New York, 1997), Sec. II, p. 193.

${ }^{98}$ H. Ohta, T. Nagaoka, K. Eriguchi, and K. Ono, Jpn. J. Appl. Phys. 48, 020225 (2009).

${ }^{99}$ T. Nagaoka, H. Ohta, K. Eriguchi, and K. Ono, Jpn. J. Appl. Phys. 48, 070219 (2009).

${ }^{100}$ N. Nakazaki, Y. Takao, K. Eriguchi, and K. Ono, Jpn. J. Appl. Phys. 53, 056201 (2014).

${ }^{101}$ T. Sakai, A. Sakai, and H. Okano, Jpn. J. Appl. Phys., Part 1 32, 3089 (1993).

${ }^{102}$ T. Ito K. Karahashi, S. Y. Kang, and S. Hamaguchi, J. Vac. Sci. Technol. A 31, 031301 (2013).

${ }^{103}$ B. A. Helmer and D. B. Graves, J. Vac. Sci. Technol. A 15, 2252 (1997).

${ }^{104}$ B. A. Helmer and D. B. Graves, J. Vac. Sci. Technol. A 16, 3502 (1998).

${ }^{105}$ N. Toyoda, N. Hagiwara, J. Matsuo, and I. Yamada, Nucl. Instrum. Methods Phys. Res., Sect. B 161-163, 980 (2000).

${ }^{106}$ D. B. Fenner, J. Hautala, L. P. Allen, T. G. Tetreault, A. Al-Jibouri, J. I. Budnick, and K. S. Jones, J. Vac. Sci. Technol. A 19, 1207 (2001). 